\title{
AN EVENT-DRIVEN APPROACH TO SERVERLESS SEISMIC IMAGING IN THE CLOUD
}

\author{
Philipp A. Witte \\ School of Computational Science and Engineering \\ Georgia Institute of Technology \\ Atlanta, GA 30308, USA \\ pwitte3@gatech.edu
}

\author{
Mathias Louboutin \\ School of Computational Science and Engineering \\ Georgia Institute of Technology \\ Atlanta, GA 30308, USA \\ mlouboutin3@gatech.edu
}

Henryk Modzelewski

Department of Earth, Ocean and Atmospheric Sciences

University of British Columbia

Vancouver, BC V6T 1Z4, Canada

hmodzelewski@eos.ubc.ca

\author{
Charles Jones \\ Osokey Ltd. \\ Henley-on-Thames, RG9 1AY, UK \\ charles@osokey.com
}

\author{
James Selvage \\ Osokey Ltd. \\ Henley-on-Thames, RG9 1AY, UK \\ james@osokey.com
}

\author{
Felix J. Herrmann \\ School of Computational Science and Engineering \\ Georgia Institute of Technology \\ Atlanta, GA 30308, USA \\ felix.herrmann@gatech.edu
}

\begin{abstract}
Adapting the cloud for high-performance computing (HPC) is a challenging task, as software for HPC applications hinges on fast network connections and is sensitive to hardware failures. Using cloud infrastructure to recreate conventional HPC clusters is therefore in many cases an infeasible solution for migrating HPC applications to the cloud. As an alternative to the generic lift and shift approach, we consider the specific application of seismic imaging and demonstrate a serverless and event-driven approach for running large-scale instances of this problem in the cloud. Instead of permanently running compute instances, our workflow is based on a serverless architecture with high throughput batch computing and eventdriven computations, in which computational resources are only running as long as they are utilized. We demonstrate that this approach is very flexible and allows for resilient and nested levels of parallelization, including domain decomposition for solving the underlying partial differential equations. While the event-driven approach introduces some overhead as computational resources are repeatedly restarted, it inherently provides resilience to instance shut-downs and allows a significant reduction of cost by avoiding idle instances, thus making the cloud a viable alternative to on-premise clusters for large-scale seismic imaging.
\end{abstract}

(C) 2019 IEEE. Personal use of this material is permitted. Permission from IEEE must be obtained for all other uses, in any current or future media, including reprinting/republishing this material for advertising or promotional purposes, creating new collective works, for resale or redistribution to servers or lists, or reuse of any copyrighted component of this work in other works. 


\section{Introduction}

Seismic imaging of the earth's subsurface is one of the most computationally expensive applications in scientific computing, as state-of-the-art imaging methods such as least-squares reverse time migration (LS-RTM), require repeatedly solving a large number of forward and adjoint wave equations during numerical optimization (e.g. [1-4]). Similar to training neural networks, the gradient computations in seismic imaging are based on backpropagation and require storage or re-computations of the state variables (i.e. of the forward modeled wavefields). Due to the large computational cost of repeatedly modeling wave propagation over many time steps using finite difference modeling, seismic imaging requires access to high-performance computing (HPC) clusters, but the high cost of acquiring and maintaining HPC cluster makes this option only viable for a small number of major energy companies [5] 6]. For this reason, cloud computing has lately emerged as a possible alternative to on-premise HPC clusters, offering many advantages such as no upfront costs, a pay-as-you-go pricing model and theoretically unlimited scalability. Outside of the HPC community, cloud computing is today widely used by many companies for general purpose computing, data storage and analysis or machine learning. Customers of cloud providers include major companies such as General Electric (GE), Comcast, Shell or Netflix, with the latter hosting their video streaming content on Amazon Web Services (AWS) [7]. Netflix' utilization of the cloud for large-scale video streaming has acted as a driver for improving the scalability of cloud tools such as object storage and event-driven computations [8], which are not available on conventional HPC environments and which we will subsequently adapt for our purposes.

However, adapting the cloud for high-performance computing applications such as seismic imaging, is not straight-forward, as numerous investigations and case studies have shown that the cloud generally cannot provide the same performance, low latency, high bandwidth and mean time between failures (MTBF) as conventional HPC clusters. An early performance analysis by Jackson [9] of a range of typical NERSC HPC applications on Amazon's Elastic Compute Cloud (EC2) found that, at the time of the comparison, applications on EC2 ran between 2.7 to 50 times slower than on a comparable HPC system due to poor network performance and that the latency was up to 400 times worse. A performance analysis by [10] using standard benchmark suites such as the HPC challenge (HPCC) supports these observations, finding that the performance on various cloud providers is in the order of one magnitude worse than to comparable HPC clusters. Other performance studies using standardized benchmarks suites, as well as domain-specific applications, similarly conclude that poor network performance severely limits the HPC capabilities of the cloud [11-17].

While communication and reliability are the strongest limiting factors in the performance of HPC applications in the cloud, several investigations [18-20] point out that embarrassingly parallel applications show in fact very good performance that is comparable to (non-virtualized) HPC environments. Similarly, performance tests on single cloud nodes and bare-metal instances using HPCC and high-performance LINPACK benchmarks [21], demonstrate good performance and scalability as well [22, 23]. These findings underline that the lift and shift approach for porting HPC applications to the cloud is unfavorable, as most HPC codes are based on highly synchronized message passing (i.e. MPI [24]) and rely on stable and fast network connections, which are not (yet) available. On the other hand, compute nodes and architectures offered by cloud computing are indeed comparable to current supercomputing systems [23] and the cloud offers a range of novel technologies such as cloud object storage or event-driven computations [25]. These technologies are not available on traditional HPC systems and make it possible to address computational bottlenecks of HPC in fundamentally new ways. Cloud object storage, such as Amazon's Simple Storage Service (S3) [26] or Google Cloud Storage [27], are based on the distribution of files to physically separated data centers and thus provide virtual unlimited scalability, as the storage system is not constrained by the size and network capacity of a fixed number of servers [28]. Successfully porting HPC applications to the cloud therefore requires a careful re-architecture of the corresponding codes and software stacks to take advantage of these technologies, while minimizing communication and idle times. This process is heavily application dependent and requires the identification of how a specific application can take advantage of specialized cloud services such as serverless compute or high throughput batch processing to mitigate resilience issues, avoid idle instances and thus minimize cost.

Based on these premises, we present a workflow for large-scale seismic imaging on AWS, which does not rely on a conventional cluster of virtual machines, but is instead based on a serverless visual workflow that takes advantage of the mathematical properties of the seismic imaging optimization problem [29]. Similar to deep learning, objective functions in seismic imaging consist of a sum of (convex) misfit functions and iterations of the associated optimization algorithms exhibit the structure of a MapReduce program [30]. The map part corresponds to computing the gradient of each element in the sum and is embarrassingly parallel to compute, but individual gradient computations are expensive as they involve solving partial differential equations (PDEs). The reduce part corresponds to the summation of the gradients and update of the model parameters and is comparatively cheap to compute, but I/O intensive. Instead of performing these steps on a cluster of permanently running compute instances, our workflow is based on specialized AWS services such as AWS Batch and Lambda functions, which are responsible for automatically launching and terminating the required computational resources [25, 31]. EC2 instances are only running as long as they are utilized and are shut down automatically as soon as computations are finished, thus preventing instances from sitting idle. This stands in contrast to alternative MapReduce cloud services, such as Amazon's Elastic Map Reduce (EMR), which is based on Apache Hadoop and relies on a cluster of permanently running EC2 instances [32, 33]. In our approach, expensive gradient computations are carried out by AWS Batch, a service for processing embarrassingly parallel workloads, but with the possibility of using (MPI-based) domain decomposition for individual solutions of partial differential equations (PDEs). The cheaper gradient summations are 
performed by Lambda functions, a service for serverless computations, in which code is run in response to events, without the need to manually provision computational resources [25].

The following section provides an overview of the mathematical problem that underlies seismic imaging and we identify possible characteristics that can be taken advantage of to avoid the aforementioned shortcomings of the cloud. In the subsequent section, we describe our seismic imaging workflow, which has been developed specifically for AWS, but the underlying services are available on other cloud platforms (Google Compute Cloud, Azure) as well. We then present a performance analysis of our workflow on a real-world seismic imaging application, using a popular subsurface benchmark model [34]. Apart from conventional scaling tests, we also consider specific cloud metrics such as resilience and cost, which, aside from the pure performance aspects like scaling and time-to-solution, are important practical considerations for HPC in the cloud. An early application of our workflow is presented in [35].

\section{Problem Overview}

Seismic imaging and parameter estimation are a set of computationally challenging inverse problems with high practical importance, as they are today widely used in the oil and gas $(\mathrm{O} \& \mathrm{G})$ industry for geophysical exploration, as well as for monitoring geohazards. In the context of exploration, seismic imaging can significantly increase the success rate of drilling into reservoirs, thus reducing both cost and environmental impact of resource exploration [36].

Mathematically, seismic imaging and parameter estimation are PDE-constrained optimization problems, that are typically expressed in the following (unconstrained) form [37 38]:

$$
\underset{\mathbf{m}}{\operatorname{minimize}} \Phi(\mathbf{m})=\sum_{i=1}^{n_{s}} \frac{1}{2}\left\|\mathcal{F}\left(\mathbf{m}, \mathbf{q}_{i}\right)-\mathbf{d}_{i}\right\|_{2}^{2},
$$

where $\mathcal{F}\left(\mathbf{m}, \mathbf{q}_{i}\right)$ represents the solution of the acoustic wave equation for a given set of model parameters $\mathbf{m}$. The evaluation of this operator corresponds to modeling seismic data for a given subsurface model (or image) $\mathbf{m}$ and a known source function $\mathbf{q}_{i}$ by solving a wave equation using time-domain finite-difference modeling. The vector $\mathbf{d}_{i}$ denotes the observed seismic measurements at the $i^{\text {th }}$ location of the seismic source, which is moved along the surface within the survey area (Figure 11). In essence, the goal of seismic inversion is to find a set of model parameters $\mathbf{m}$, such that the numerically modeled data matches the observed data from the seismic survey. The total number of individual source experiments $n_{s}$ for realistic surveys, i.e. the number of PDEs that have to solved for each evaluation of $\Phi(\mathbf{m})$, is quite large and lies in the range of $10^{3}$ for $2 \mathrm{D}$ surveys and $10^{5}$ for $3 \mathrm{D}$ surveys.

Seismic inverse problems of this form are typically solved with gradient-based optimization algorithms such as (stochastic) gradient descent, (Gauss-) Newton methods, sparsity-promoting minimization or constrained optimization (e.g. [39, 40]) and therefore involve computing the gradient of Equation 1 for all or a subset of indices $i$. The gradient of the objective function is given by:

$$
\mathbf{g}=\sum_{i=1}^{n_{s}} \mathbf{J}^{\top}\left(\mathcal{F}\left(\mathbf{m}, \mathbf{q}_{i}\right)-\mathbf{d}_{i}\right),
$$

where the linear operator $\mathbf{J}=\frac{\partial \mathcal{F}\left(\mathbf{m}, \mathbf{q}_{i}\right)}{\partial \mathbf{m}}$ is the partial derivative of the forward modeling operator with respect to the model parameters $\mathbf{m}$ and $T$ denotes the matrix transpose. Both the objective function, as well as the gradient exhibit a sum structure over the source indices and are embarrassingly parallel to compute. Evaluating the objective function and computing the gradient are therefore instances of a MapReduce program [30], as they involve the parallel computation and subsequent summation of elements of the sum. However, computing the gradient for a single index $i$ involves solving two PDEs, namely a forward wave equation and an adjoint (linearized) wave equation (denoted as a multiplication with $\mathbf{J}^{\top}$ ). For realistically sized 3D problems, the discretized model in which wave propagation is modeled has up to $10^{9}$ variables and modeling has to be performed for several thousand time steps. The number of time steps is determined by the time stepping interval and depends on the wave speed and the temporal frequency of the data and increases significantly as these properties change [41]. The observed seismic data $\mathbf{d}_{i}\left(i=1, \ldots, n_{s}\right)$ is typically in the range of several terabytes and a single element of the data (a seismic shot record) ranges from several mega- to gigabytes.

The problem structure of equation 1 is very similar to deep learning and the parallels between convolutional neural networks and PDEs have lately attracted strong attention [42]. As in deep learning, computing the gradient of the objective function (equation 2) is based on backpropagation and in principle requires storing the state variables of the forward problem. However, in any realistic setting the wavefields are too big to be stored in memory and therefore need to be written to secondary storage devices or recomputed from a subset of checkpoints [43]. Alternatively, domain decomposition can be used to reduce the domain size per compute node such that the forward wavefields fit in memory, or time-to frequency conversion methods can be employed to compute gradients in the frequency domain [4, 44]. In either case, computing the gradient for a given index $i$ is expensive both in terms of necessary floating point operations, memory and IO and requires highly optimized finite-difference modeling codes for solving the underlying wave equations. Typical computation times of a single (3D-domain) gradient $\mathbf{g}_{i}$ (i.e. one element of the sum) are in the range of minutes to hours, depending on the domain size and the complexity of the wave simulator, and the computations have to be carried out for a large number of source locations and iterations. 


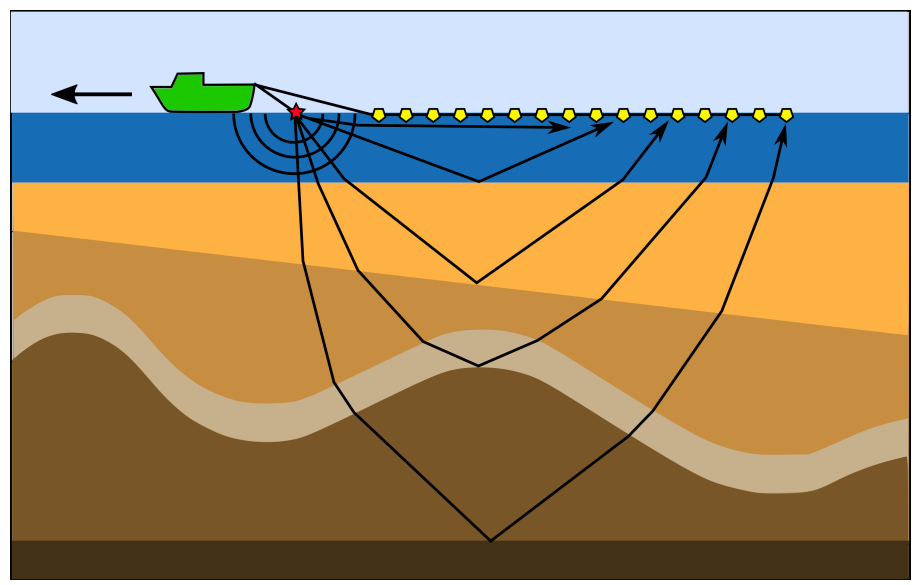

Figure 1: A two-dimensional depiction of marine seismic data acquisition. A vessel fires a seismic source and excites acoustic waves that travel through the subsurface. Waves are reflected and refracted at geological interfaces and travel back to the surface, where they are recorded by an array of seismic receivers that are towed behind the vessel. The receivers measure pressure changes in the water as a function of time and receiver number for approximately 10 seconds, after which the process is repeated. A typical seismic survey consists of several thousand of these individual source experiments, during which the vessel moves across the survey area.

The high computational cost of seismic modeling, in combination with the complexity of implementing optimization algorithms to solve equation 1 leads to enormously complex inversion codes, which have to run efficiently on large-scale HPC clusters. A large amount of effort goes into implementing fast and scalable wave equation solvers [45. 47], as well as into frameworks for solving the associated inverse problem [48 51]. Codes for seismic inversion are typically based on message passing and use MPI to parallelize the loop of the source indices (equation 11. Furthermore, a nested parallelization is oftentimes used to apply domain-decomposition or multi-threading to individual PDE solves. The reliance of seismic inversion codes on MPI to implement an embarrassingly parallel loop is disadvantageous in the cloud, where the mean-time-between failures (MTBF) is much shorter than on HPC systems [9] and instances using spot pricing can be arbitrarily shut down at any given time [52]. Another important aspect is that the computation time of individual gradients can vary significantly and cause load imbalances and large idle times, which is problematic in the cloud, where users are billed for running instances by the second, regardless of whether the instances are in use or idle. For these reasons, we present an alternative approach for seismic imaging in the cloud based on batch processing and event-driven computations.

\section{Event-driven seismic imaging on AWS}

\subsection{Workflow}

Optimization algorithms for minimizing equation 1 essentially consists of three steps. First, the elements of the gradient $\mathbf{g}_{i}$ are computed in parallel for all or a subset of indices $i \in n_{s}$, which corresponds to the map part of a MapReduce program. The number of indices for which the objective is evaluated defines the batch size of the gradient. The subsequent reduce part consists of summing these elements into a single array and using them to update the unknown model/image according to the rule of the respective optimization algorithm (Algorithm 1). Optimization algorithms that fit into this general framework include variations of stochastic/full gradient descent (GD), such as Nesterov's accelerated GD [53] or Adam [54], as well as the nonlinear conjugate gradient method [55], projected GD or iterative soft thresholding [56]. Conventionally, these algorithms are implemented as a single program and the gradient computations for seismic imaging are parallelized using message passing. Running MPI-based programs of this structure in the cloud (specifically on AWS), require that users request a set of EC2 instances and establish a network connection between all workers [57]. Tools like StarCluster [58] or AWS HPC [59] facilitate the process of setting up a cluster and even allow adding or removing instances to a running cluster. However, adding or dropping instances/nodes during the execution of an MPI program is not easily possible, so the number of instances has to stay constant during the entire length of the program execution, which, in the case of seismic inversion, can range from several days to weeks. This makes this approach not only prone to resilience issues, but it can result in significant cost overhead, if workloads are unevenly distributed and instances are temporarily idle. 


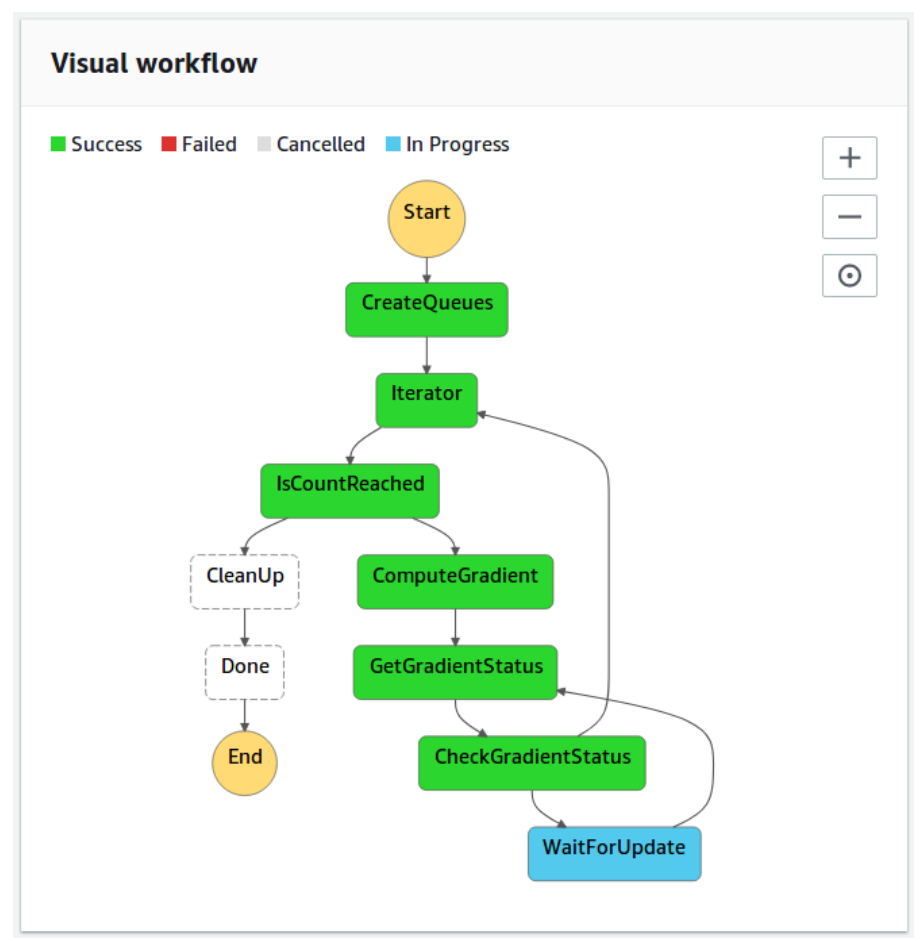

Figure 2: A generic seismic imaging algorithm, expressed as a serverless visual workflow using AWS Step Functions. The workflow consists as a collection of states, which are used to implement an iterative optimization loop. Each iteration involves computing the gradient of Equation 1 using AWS Batch, as well an updating the optimization variable (i.e. the seismic image).

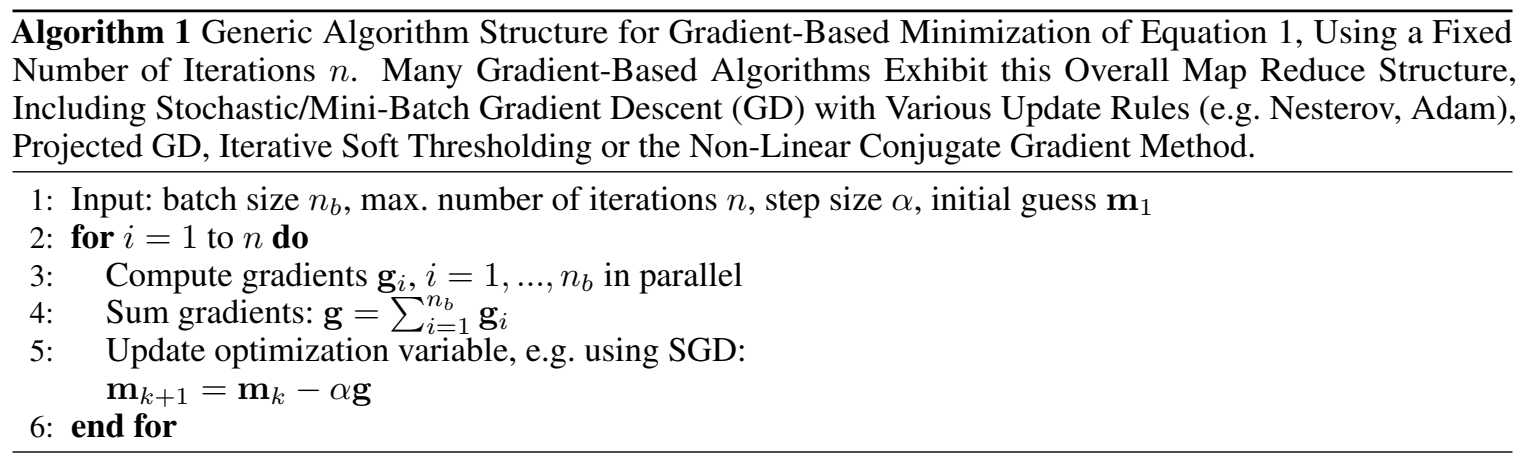

Instead of implementing and running optimization algorithms for seismic inverse problems as a single program that runs on a cluster of EC2 instances, we express the steps of a generic optimization algorithm through AWS Step Functions (Figure 2) and deploy its individual components through a range of specialized AWS services [60]. Step functions allow the description of an algorithm as a collection of states and their relationship to each other using the JavaScript Object Notation (JSON). From the JSON definition of a workflow, AWS renders an interactive visual workflow in the web browser, as shown in Figure 2 For our purpose, we use Step Functions to implement our iterative loop [61], during which we compute and sum the gradients, and use them to update the seismic image. We choose Step Functions to express our algorithm, as they allow composing different AWS Services such as AWS Batch and Lambda functions into a single workflow, thus making it possible to leverage preexisiting AWS services and to combine them into a single application. Another important aspect of Step Functions is that the execution of the workflow itself is managed by AWS and does not require running any EC2 instances, which is why we refer to this approach as serverless. During execution time, AWS automatically progresses the workflow from one state to the next and users are only billed for transitions between states, but the cost is negligible compared to the cost of running EC2 instances ( $0.025 \$$ per 1,000 state transitions).

States can be simple if-statements such as the IsCountReached state, which keeps track of the iteration number and terminates the workflow after a specified number of iterations, but states can also be used invoke other AWS services. Specifically, states can be used to invoke AWS Lambda functions to carry out serverless computations. Lambda functions 
allow users to run code in response to events, such as invocations through AWS Step Functions, and automatically assign the required amount of computational resources to run the code. Billing is based on the execution time of the code and the amount of used memory. Compared to EC2 instances, Lambda functions have a much shorter startup time in the range of milliseconds rather than minutes, but they are limited to $3 \mathrm{~GB}$ of memory and an execution time of 15 minutes. As such, Lambda functions themselves are not suitable for carrying out the gradient computations, but they can be used to manage other AWS services. In our workflow, we use Lambda functions invoked by the ComputeGradient state (Figure 2) to launch AWS Batch jobs for computing the gradients. During the gradient computation, which can take up to several hours, the Step Functions check in a user-defined interval if the full gradient has been computed, before advancing the workflow to the next state. The WaitForGradient state pauses the workflow for a specified amount of time, during which no additional computational resources are running other than the AWS Batch job itself.

\subsection{Computing the gradient}

The gradient computations (equation 2) are the major workload of seismic inversion, as they involve solving forward and adjoint wave equations, but the embarrassingly parallel structure of the problem lends itself to high-throughput batch computing. On AWS, embarrassingly parallel workloads can be processed with AWS Batch, a service for scheduling and running parallel containerized workloads on EC2 instances [31]. Parallel workloads, such as computing a gradient of a given batch size, are submitted to a batch queue and AWS Batch automatically launches the required EC2 instances to process the workload from the queue. Each job from the queue runs on an individual instance or set of instances, with no communication being possible between individual jobs.

In our workflow, we use the Lambda function invoked by the ComputeGradient state (Figure 2) to submit the gradient computations to an AWS Batch queue. Each element of the gradient $\mathbf{g}_{i}$ corresponds to an individual job in the queue and is run by AWS Batch as a separate Docker container [62]. Every container computes the gradient for its respective source index $i$ and writes its resulting gradient to an S3 bucket (Figure 3], Amazon's cloud object storage system [26]. The gradients computed by our workflow are one-dimensional numpy arrays of the size of the vectorized seismic image and are stored in $\mathrm{S} 3$ as so-called objects [63]. Once an individual gradient $\mathbf{g}_{i}$ has been computed, the underlying EC2 instance is shut down automatically by AWS Batch, thus preventing EC2 instances from idling. Since no communication between jobs is possible, the summation of the individual gradients is implemented separately using AWS Lambda functions. For this purpose, each jobs also sends its S3 object identifier to a message queue (SQS) [64], which automatically invokes the reduction stage (Figure 4). For the gradient computations, each worker has to download the observed seismic data of its respective source index from S3 and the resulting gradient has to be uploaded to S3 as well. The bandwidth with which objects are up- and downloaded is only limited by the network bandwidth of the EC2 instances and ranges from 10 to 100 Gbps [65]. Notably, cloud object storage such as S3 has no limit regarding the number of workers that can simultaneously read and write objects, as data is (redundantly) distributed among physically separated data centers, thus providing essentially unlimited IO scalability [26].

AWS Batch runs jobs from its queue as separate containers on a set of EC2 instances, so the source code of the application has to be prepared as a Docker container. Containerization facilitates portability and has the advantage that users have full control over managing dependencies and packages. Our Docker image contains the code for solving acoustic wave equations to compute gradients of a respective seismic source location. Since this is the most computational intensive part of our workflow, it is important that the wave equation solver is optimized for performance, but is also implemented in a programming language that allows interfacing other AWS services such as S3 or SQS. In our workflow, we use the domain-specific language compiler called Devito for implementing and solving the underlying wave equations using timedomain finite-difference modeling [47, 66. Devito is implemented in Python and provides an application programming interface (API) for implementing forward and adjoint wave equations as high-level symbolic expressions based on the SymPy package [67]. During runtime, the Devito compiler applies a series of performance optimizations to the symbolic operators, such as reductions of the operation count, loop transformations, and introduction of parallelism [66]. Devito then generates optimized finite-difference stencil code in $\mathrm{C}$ from the symbolic Python expressions and dynamically compiles and runs it. Devito supports both multi-threading using OpenMP, as well as generating code for MPI-based domain decomposition. Its high-level API allows expressing wave equations of arbitrary stencil orders or various physical representations without having to implement and optimize low-level stencil codes by hand. Furthermore, Devito includes various possibilities for backpropagation, such as optimal checkpointing or on-the-fly Fourier transforms [35, 68]. The complexity of implementing highly optimized and parallel wave equation solvers is therefore abstracted and vertically integrated into the AWS workflow.

By default, AWS Batch runs the container of each job on a single EC2 instance, but recently AWS introduced the possibility to run multi-node batch computing jobs [69]. Thus, individual jobs from the queue can be computed on a cluster of EC2 instances and the corresponding Docker containers can communicate via the AWS network. As for single-instance jobs, AWS Batch automatically requests and terminates the EC2 instances on which the Docker containers are deployed. In the context of seismic imaging and inversion, multi-node batch jobs enable nested levels of parallelization, as we can use AWS Batch to parallelize the sum of the source indices, while using MPI-based domain decomposition and/or multi-threading for solving the underlying wave equations. This provides a large amount of flexibility in regard of the computational strategy for performing backpropagation and how to address the storage of the state variables. AWS Batch allows to scale horizontally, by increasing the number of EC2 instances of multi-node jobs, but also enables vertical scaling by 


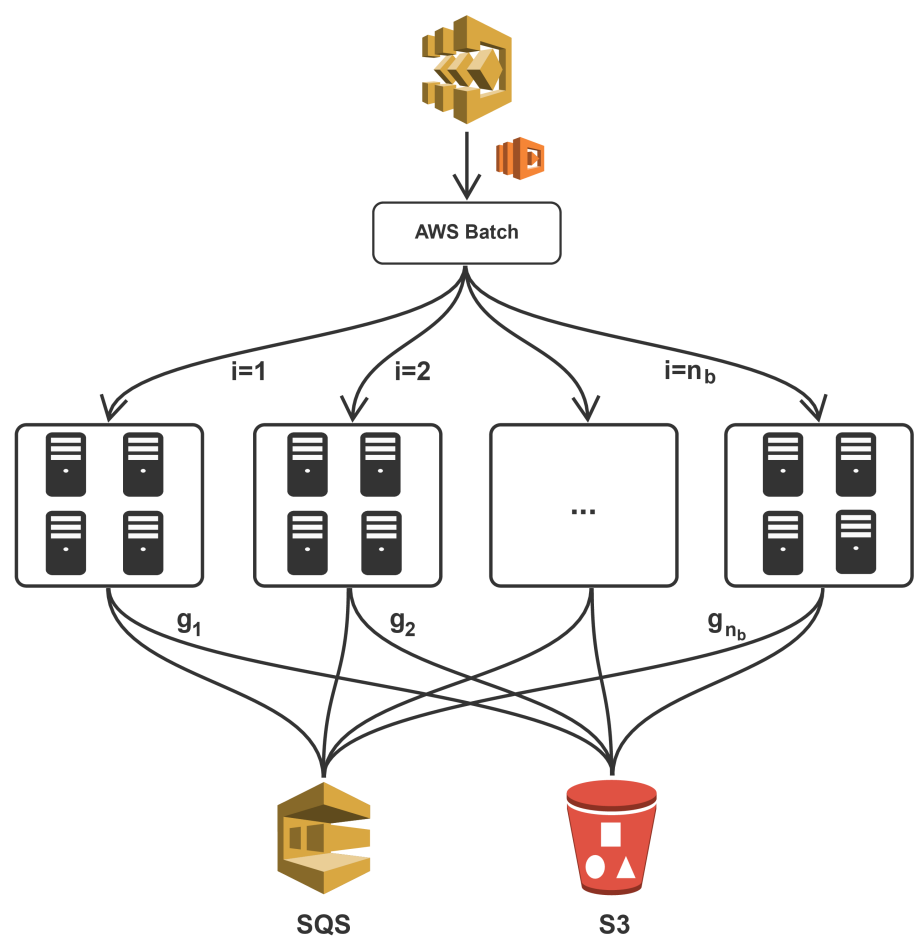

Figure 3: The gradients of the LS-RTM objective function are computed as an embarrassingly parallel workload using AWS Batch. This process is automatically invoked by the AWS Step Functions (Figure 2) during each iteration of the workflow. The gradients of individual source locations are computed as separate jobs on either a single or multiple EC2 instances. Communication is only possible between instances of a single job, but not between separate jobs. The resulting gradients are saved in $\mathrm{S} 3$ and the respective object names are sent to an SQS queue to invoke the gradient summation.

adding additional cores and/or memory to single instances. In our performance analysis, we compare and evaluate different strategies for computing gradients with Devito regarding scaling, costs and turnaround time.

\subsection{Gradient reduction}

Every computed gradient is written by its respective container to an S3 bucket, as no communication between individual jobs is possible. Even if all gradients in the job queue are computed by AWS Batch in parallel at the same time, we found that the computation time of individual gradients typically varies considerably (up to 10 percent), due to varying network performance or instance capacity. Furthermore, we found that the startup time of the underlying EC2 instances itself is highly variable as well, so jobs in the queue are usually not all started at the same time. Gradients therefore arrive in the bucket over a large time interval during the batch job. For the gradient reduction step, i.e. the summation of all gradients into a single array, we take advantage of the varying time-to-solutions by implementing an event-driven gradient summation using Lambda functions. In this approach, the gradient summation is not performed by as single worker or the master process who has to wait until all gradients have been computed, but instead summations are carried out by Lambda functions in response to gradients being written to S3. The event-driven summation is therefore started as soon as the first two gradients have been computed.

The event-driven gradient summation is automatically invoked through SQS messages, which are sent by the AWS Batch workers that have completed their computations and have saved their respective gradient to S3. Before being shut down, every batch worker sends a message with the corresponding S3 object name to an AWS SQS queue, in which all object names are collected (Figure 4). Sending messages to SQS invokes AWS Lambda functions that read up to 10 messages at a time from the queue. Every invoked Lambda function that contains at least two messages, i.e. two object names, reads the corresponding arrays from S3, sums them into a single array, and writes the array as a new object back to S3. The new object name is sent to the SQS queue, while the previous objects and objects names are removed from the queue and S3. The process is repeated recursively until all $n_{b}$ gradients have been summed into a single array, with $n_{b}$ being the batch size for which the gradient is computed. The gradient summation is implemented in Python, which is one of the languages supported by AWS Lambda [25]. SQS guarantees that all messages are delivered at least once to the subscribing Lambda functions, thus ensuring that no gradients are lost in the summation process [64]. 


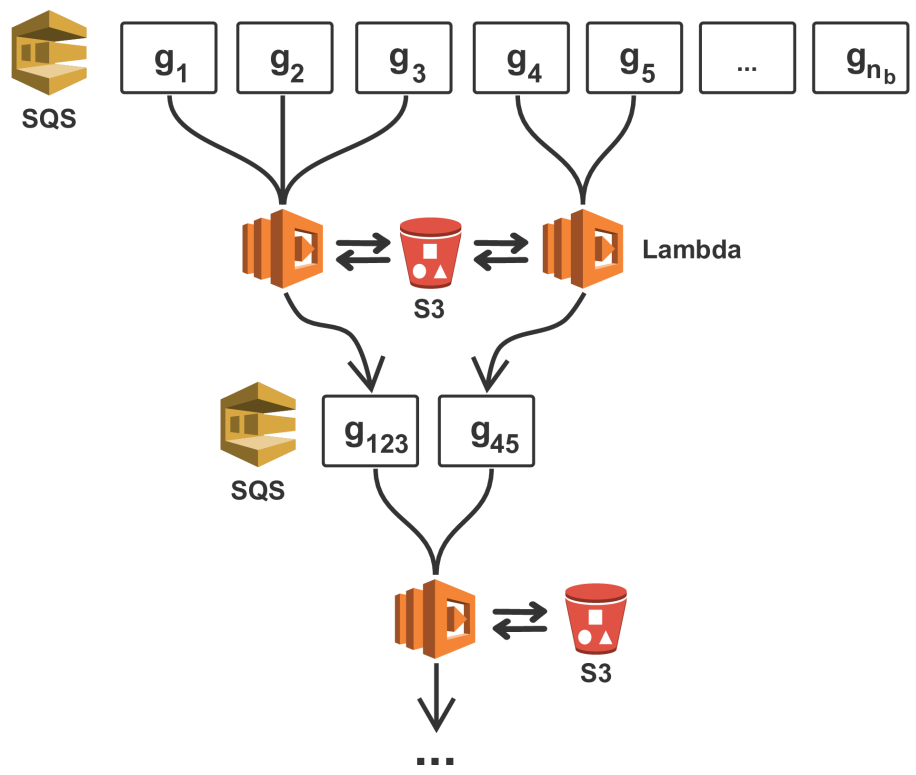

Figure 4: Event-driven gradient summation using AWS Lambda functions. An SQS message queue collects the object names of all gradients that are currently stored in S3 and automatically invokes Lambda functions that stream up to 10 files from S3. Each Lambda function sums the respective gradients, writes the result back to $\mathrm{S} 3$ and sends the new object name to the SQS queue. The process is repeated until all gradients have been summed into a single $\mathrm{S} 3$ object. SQS has a guaranteed at-least-once delivery of messages to ensure that no objects are lost in the summation.

Since Lambda functions are limited to 3 GB of memory, it is not always possible to read the full gradient objects from S3. Gradients that exceed Lambda's available memory are therefore streamed from S3 using appropriate buffer sizes and are re-uploaded to S3 using the multipart_upload functions of the S3 Python interface [70]. As the execution time of Lambda functions is furthermore limited to 15 minutes, the bandwidth of S3 is not sufficient to stream and re-upload objects that exceed a certain size within a single Lambda invocation. For this case, we include the possibility that the workers of the AWS Batch job split the computed gradients into smaller chunks that are saved separately in S3, with the respective objects names being sent to multiple SQS queues. The gradient summation is then performed in chunks by separate queues and Lambda functions. The CreateQueues task of our Step Functions workflow (Figure 2) automatically creates the required number of queues before starting the optimization loop and the CleanUp state removes them after the final iteration.

The advantage of the event-based gradient reduction is that that the summation is executed asynchronously, as soon as at least two S3 objects containing gradients are available, while other batch jobs are still running. Therefore, by the time the last batch worker finishes the computation of its respective gradient, all remaining gradients have already been summed into a single object, or at least a small number of objects. Furthermore, summing files of a single queue happens in parallel (if enough messages are in the queue), as multiple Lambda functions can be invoked at the same time. Furthermore, splitting the gradients itself into chunks that are processed by separate queues leads to an additional layer of parallelism. In comparison to a fixed cluster of EC2 instances, the event-driven gradient summation using Lambda function also takes advantage of the fact that the summation of arrays is computationally considerably cheaper than solving wave equations and therefore does not require to be carried out on the expensive EC2 instances used for the PDE solves.

\subsection{Variable update}

Once the gradients have been computed and summed into a single array that is stored as an S3 object, the gradient is used to update the optimization variables of equation 1. i.e. the seismic image or subsurface parameters such as velocity. Depending on the specific objective function and optimization algorithm, this can range from simple operations like multiplications with a scalars (gradient descent) to more computational expensive operations such as sparsity promotion or applying constraints [71]. Updates that use entry-wise operations only and are cheap to compute such as multiplications with scalars or soft-thresholding, can be applied directly by the Lambda functions in the final step of the gradient summation. I.e. the Lambda function that sums the final two gradients, also streams the optimization variable of the current iteration from S3, uses the gradient to update it and directly writes the updated variable back to S3.

Many algorithms require access to the full optimization variable and gradient, such as Quasi-Newton methods and other algorithms that need to compute gradient norms. In this case, the variable update is too expensive and memory intensive to 


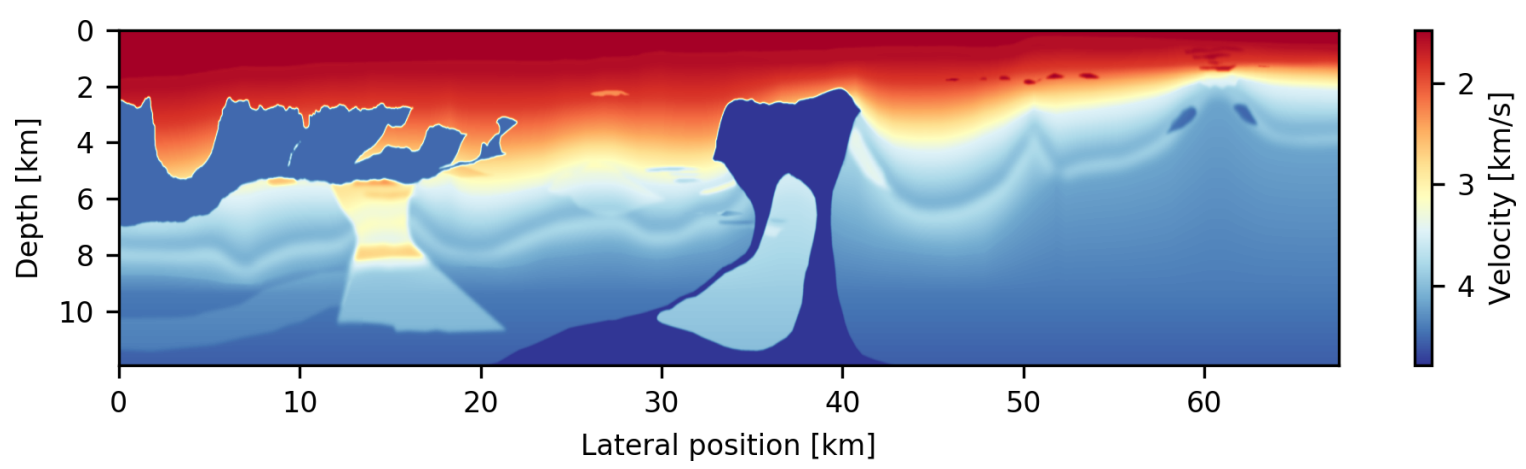

Figure 5: The BP 2004 benchmark model, a 2D subsurface velocity model for development and testing of algorithms for seismic imaging and parameter estimation [34]. This model and the corresponding seismic data set are used in our performance analysis. The velocity model and the unknown image have dimensions of $1,911 \times 10,789$ grid points, a total of 20.1 million unknown parameters.

be carried out by Lambda functions and has to be submitted to AWS Batch as a single job, which is then executed on a larger EC2 instance. This can be accomplished by adding an extra state such as UpdateVariable to our Step Functions workflow. However, to keep matters simple, we only consider a simple stochastic gradient descent example with a fixed step size in our performance analysis, which is computed by the Lambda functions after summing the final two gradients [72]. The CheckGradientStatus state of our AWS Step Functions advances the workflow to the next iteration, once the updated image (or summed gradient) has been written to S3. The workflow shown in Figure 2 terminates the optimization loop after a predefined number of iterations (i.e. epochs), but other termination criteria based on gradient norms or function values are possible too and can be realized by modifying the IsCountReached state. The update of the optimization variable concludes a single iteration of our workflow, whose performance we will now analyze in the subsequent sections.

\section{Performance analysis}

In our performance analysis, we are interested in the performance of our workflow on a real-world seismic imaging application regarding scalability, cost and turn-around time, as well as the computational benefits and overhead introduced by our event-driven approach. We conduct our analysis on a popular 2D subsurface velocity model (Figure 5), called the 2004 BP velocity estimation benchmark model [34]. This model was originally created for analyzing seismic processing or inversion algorithms, but as the model represents a typical large-scale 2D workload, we consider this model for our following performance analysis. The seismic data set of this model contains 1,348 seismic source locations and corresponding observations $\mathbf{d}_{i}(i=1, \ldots, 1,348)$. The (unknown) seismic image has dimensions of $1,911 \times 10,789$ grid points, i.e. a total of almost 21 million parameters. An overview of all grid parameters, as well as the dimensions of the seismic data are presented in Table 1 of the Appendix.

\subsection{Weak scaling}

In our first performance test, we analyze the weak scaling behavior of our workflow by varying the batch size (i.e. the number of source locations) for which the gradient of the LS-RTM objective function (Equation 1) is computed. For this test, we perform a single iteration of stochastic gradient descent (SGD) using our workflow and measure the timeto-solution as a function of the batch size. The workload per instance, i.e. per parallel worker, is fixed to one gradient. The total workload for a specified batch size is submitted to AWS Batch as a so-called array job, where each array entry corresponds to a single gradient $\mathbf{g}_{i}$. AWS Batch launches one EC2 instance per array entry (i.e. per gradient), runs the respective container on the instance and then terminates the instance afterwards. If a sufficient amount of instances are available, AWS Batch will theoretically launch all containers of the array job instantaneously and run the full workload in parallel, but as we will see in the experiment, this is in practice not necessarily the case.

In the experiment, we measure the time-to-solution for performing a single iteration of our workflow, i.e. one stochastic gradient descent update. We exclude the setup time of the SQS queues, which is the first step of our workflow (Figure 2), as this process only has to be performed once, prior to the first iteration. Therefore, each run involves the following steps:

1. A Lambda function submits the AWS Batch job for specified batch size $n_{b}$ (Figure 3 )

2. Compute gradients $\mathbf{g}_{i}\left(i=1, \ldots, n_{b}\right)$ in parallel (Figure 3)

3. Lambda functions sum the gradients (Figure 4): $\mathbf{g}=\sum_{i=1}^{n_{b}} \mathbf{g}_{i}$

4. A Lambda function performs the SGD update of the image: $\mathbf{x}=\mathbf{x}-\alpha \mathbf{g}$ 
We define the time-to-solution as the the time interval between the submission of the AWS Batch job by a Lambda function (step 1) and the time stamp of the S3 object containing the updated image (step 4). This time interval represents a complete iteration of our workflow.

The computations of the gradients are performed on $\mathrm{m} 4.4 \mathrm{xlarge}$ instances and the number of threads per instance is fixed to 8 , which is the number of physical cores that is available on the instance. The $\mathrm{m} 4$ instance is a general purpose EC2 instance and we chose the instance size (4xlarge) such that we are able to store the wavefields for backpropagation in memory. The workload for each batch worker consists of solving a forward wave equation to model the predicted seismic data and an adjoint wave equation to backpropagate the data residual and to compute the gradient. For this and all remaining experiments, we use the acoustic isotropic wave equation with a second order finite difference (FD) discretization in time and 8th order in space. We model wave propagation for 12 seconds, which is the recording length of the seismic data. The time stepping interval is given by the Courant-Friedrichs-Lewy condition with $0.55 \mathrm{~ms}$, resulting in 21,889 time steps. Since it is not possible for the waves to propagate through the whole domain within this time interval, we restrict the modeling grid to a size of $1,911 \times 4,001$ grid points around the current source location. After modeling, each gradient is extended back to the full model size $(1,911 \times 10,789$ grid points). A detailed description of the setup parameters and utilized software and hardware is provided in the appendix (Table 2). The dimensions of this example represent a large-scale 2D example, but all components of our workflow are agnostic to the number of physical dimensions and are implemented for three-dimensional domains as well. The decision to limit the examples to a 2D model was purely made from a financial viewpoint and to make the results reproducible in a reasonable amount of time.

The timings ranging from a batch size of 1 to 128 are displayed in Figure $6 \mathrm{a}$. The batch size corresponds to the number of parallel EC2 instances on which the jobs are executed. The time-to-solution consists of three components that make up the full runtime of each job:

1. The average time for AWS Batch to request and launch the EC2 instances and to start the Docker containers on those instances.

2. The runtime of the containers

3. The additional gradient reduction and image update time, which is given by the time interval between the termination of the AWS Batch job and the time stamp of the updated variable.

The sum of these components makes up the time-to-solution as shown in Figure 6a and each component is furthermore plotted separately in Figures 6b to 6d All timings are the arithmetic mean over three individual runs and the standard deviation is indicated by the error bars. The container runtimes of Figure 6c are the arithmetic mean of the individual container runtimes on each instance (varying from 1 to 128). The average container runtime is proportional to the cost of computing one individual gradient and is given by the container runtime times the price of the $\mathrm{m} 4.4 \mathrm{xlarge}$ instance, which was $\$ 0.2748$ per hour at the time of testing. AWS Batch automatically launches and terminates the EC2 instance on which each gradient is computed and the user only pays for utilized EC2 time. No extra charges occurs for AWS Batch itself, i.e. for scheduling and launching the batch job.

The timings indicate that the time-to-solution generally grows as the batch size, and therefore the number of containers per job, increases (Figure 6a). A close up inspection of the individual components that make up the total time-to-solution shows that this is mostly due to the increase of the startup time, i.e. the average time it takes AWS Batch to schedule and launch the EC2 instances of each job (Figure 6b). We monitored the status of the EC2 instances during the job execution and found that AWS Batch does generally not start all instances of the array job at the same time, but instead in several stages, over the course of 1 to 3 minutes. The exact startup time depends on the batch size and therefore on the number of instances that need to be launched, but also on the availability of the instance within the AWS region. The combination of these factors lead to an increase of the average startup time for an increasing batch size, but also to a large variance of the startup time between individual runs. Unfortunately, the user has no control over the startup time, but it is important to consider that no cost is incurred during this time period, as no EC2 instances are running while the individual containers remain in the queue.

The average container runtime, i.e. the average computation time of a single gradient within the batch, is fairly stable as the batch size increases (Figure 6c). This observation is consistent with the fact that each container of an AWS Batch array job runs as an individual Docker container and is therefore independent of the batch size. The container runtime increases only slightly for larger batch sizes and we observe a large variance in some of the container runtimes (specifically for a batch size of 8). This variance stems from the fact that users do not have exclusive access to the EC2 instances on which the containers are deployed. Specifically, our containers run on $\mathrm{m} 4.4 \mathrm{xlarge}$ instances, which have 8 cores (16 virtual CPUs) and 64 GB of memory. In practice, AWS deploys these instances on larger physical nodes and multiple EC2 instances (of various users) can run on the same node. We hypothesize that a larger batch size increases the chance of containers being deployed to a compute node that runs at full capacity, thus slightly increasing the average container runtime, as user do not have exclusive access to the full network capacity or memory bandwidth.

Finally, we also observe an increase in the additional gradient reduction time, i.e. the interval between the S3 timestamps of the final computed gradient $\mathbf{g}_{i}$ and the updated image $\mathbf{x}$. The batch size corresponds to the number of gradients that have to be summed before the gradient can be used to update the image.The event-driven gradient reduction invokes the summation process as soon as the first gradients are written to S3, so most gradients are already summed by the time 


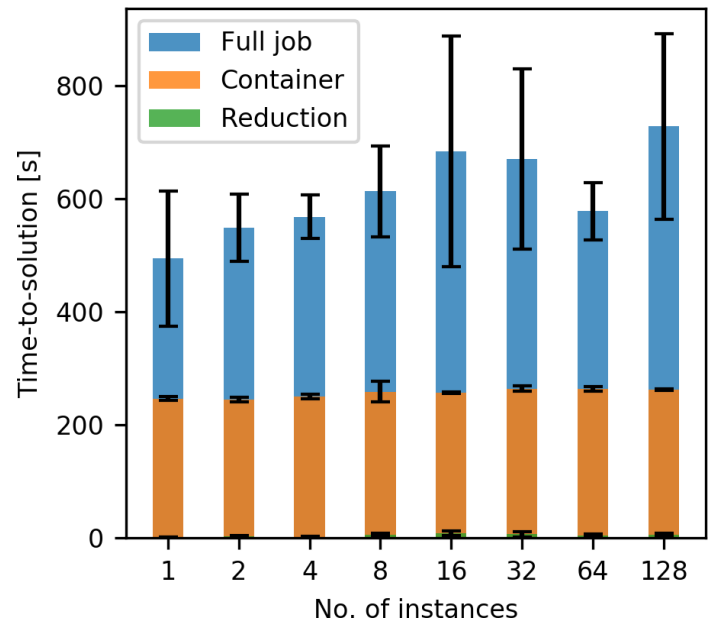

(a)

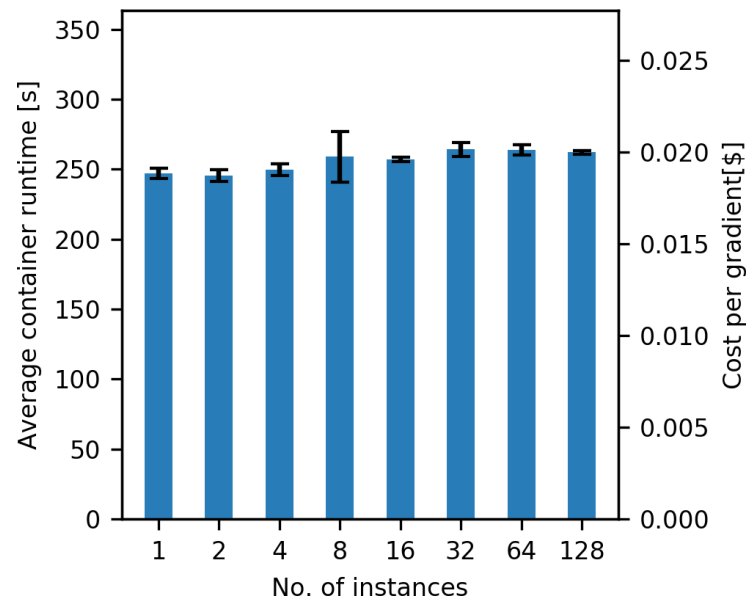

(c)

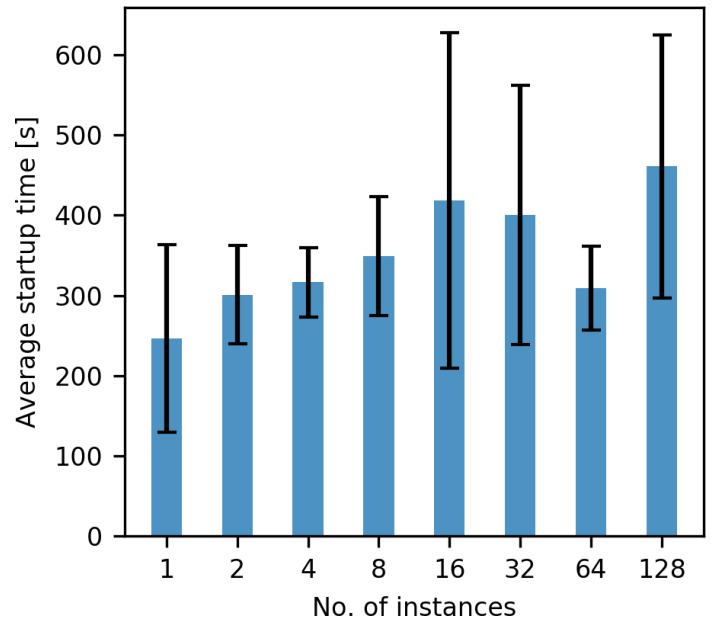

(b)

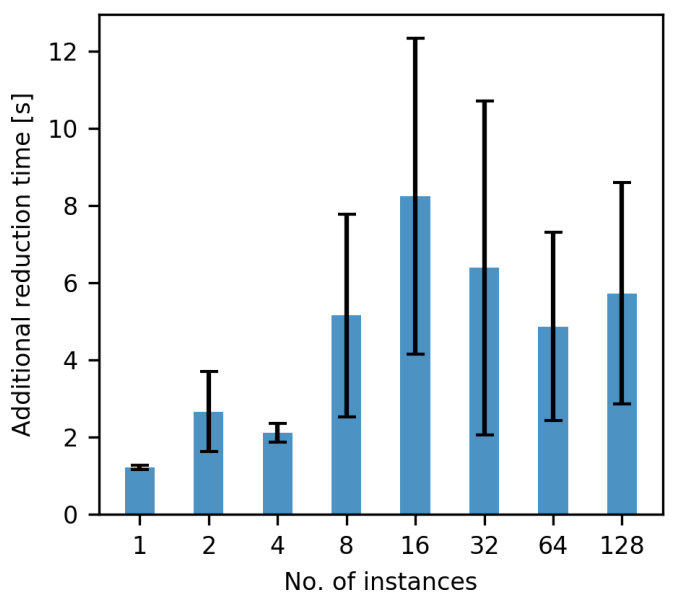

(d)

Figure 6: Weak scaling results for performing a single iteration of stochastic gradient as a function of the batch size for which the gradient is computed (a). The gradient is computed as an AWS Batch job with an increasing number of parallel EC2 instances, while the gradient summation and the variable update are performed by Lambda functions. The total time-to-solution (a) consists of the average time it takes AWS Batch to request and start the EC2 instances (b), the average runtime of the containers (c) and the additional reduction time (d), i.e. the time difference between the final gradient of the respective batch and the updated image. All timings are the arithmetic mean over three runs, with the error bars representing the standard deviation.

the final worker finishes its gradient computation. For the event-driven gradient summation, the variance of the startup and container runtime is therefore advantageous, as it allows the summation to happen asynchronously. However, in our example, the time interval between the first two gradients being written to S3 (thus invoking the gradient reduction) and the final gradient being computed, does not appear to be large enough to complete the summation of all gradients. Specifically, we see an increase in the reduction time from a batch size of 4 to 8 , after which the additional reduction is mostly constant, but again with a large variance. This variance is due to a non-deterministic component of our event-based gradient summation, resulting from a limitation of AWS Lambda. While users can specify a maximum number of messages that Lambda functions read from an SQS queue, it is not possible to force Lambda to read a minimum amount of two messages, resulting in most Lambda functions reading only a single message (i.e. one object name) from the queue. Since we need at least two messages to sum the corresponding gradients, we return the message to the queue and wait for a Lambda invocation with more than one message. The user has no control over this process and sometimes it takes several attempts until a Lambda function with multiple messages is invoked. The likelihood of this happening increases with a growing batch size, since a larger number of gradients need to be summed, which explains the increase of the reduction time and variance in Figure 6d. 


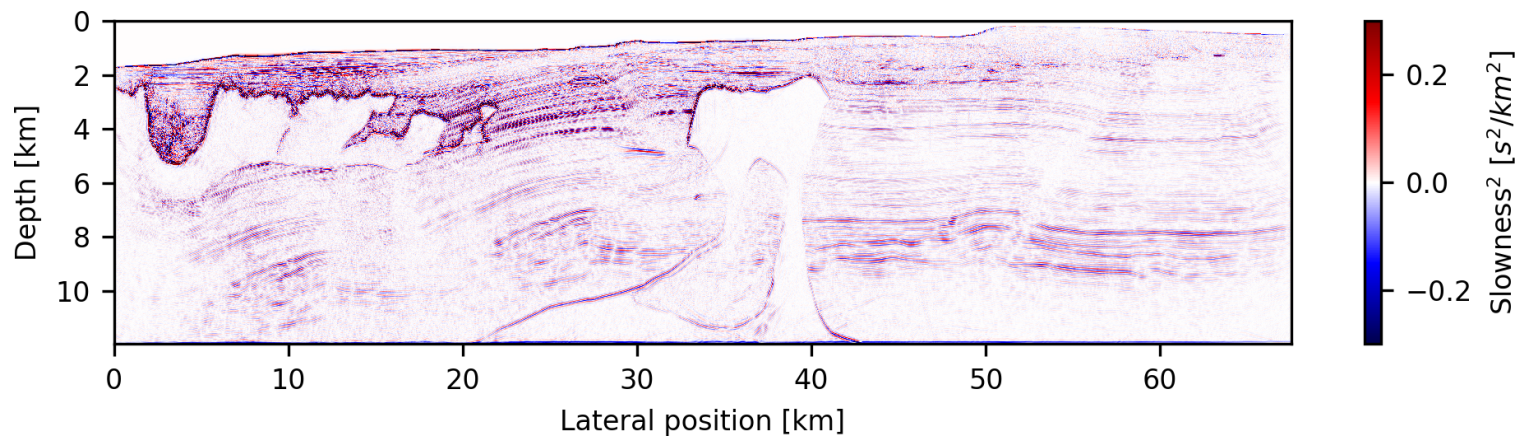

Figure 7: Final seismic image after 30 iterations of stochastic gradient descent and a batch size of 80 , which corresponds to approximately two passes through the data set (i.e. two epochs).

Overall, the gradient summation and variable update finishes within a few seconds after the last gradient is computed and the additional reduction time is small compared to the full time-to-solution and to the pure computation time of the gradients. In our example, the startup time (Figure 6b) takes up the majority of the time-to-solution (Figure 6a), as it lies in the range of a few minutes and is in fact longer than the average container runtime of each worker (Figure $6 \mathrm{c}$ ). However, the startup time is independent of the runtime of the containers, so the ratio of the startup time to the container runtime improves as the workload per container increases. Furthermore, the cost of the batch job only depends on the container runtime and the batch size, but not on the startup time or reduction time. The cost for summing the gradients is given by the cumulative runtime of the Lambda functions, but is negligible compared to the EC2 cost for computing the gradients. At the time of the example, the cost for Lambda functions was $\$ 2 \cdot 10^{-7}$ per request and $\$ 1.6 \cdot 10^{-5}$ per used GB-second. Figure 7 shows the final seismic image that is obtained after running our workflow for 30 iterations and a batch size of 80 , which corresponds to 1.8 epochs. The source locations in each iteration are chosen from a uniform random distribution and and after the final iteration, each data sample (i.e. seismic shot record) has been, in expectation, used 1.8 times. In this example, every gradient was computed by AWS Batch on a single instance and a fixed number of threads, but in the subsequent section we analyze the scaling of runtime and cost as a function of the number of cores and EC2 instances. Furthermore, we will analyze in a subsequent example how the cost of running the gradient computations with AWS Batch compares to performing those computations on a fixed cluster of EC2 instances.

\subsection{Strong scaling}

In the following set of experiments, we analyze the strong scaling behavior of our workflow for an individual gradient calculation, i.e. a gradient for a batch size of 1 . For this, we consider a single gradient computation using AWS Batch and measure the runtime as a function of either the number of threads or the number of instances in the context of MPI-based domain decomposition. In the first experiment, we evaluate the vertical scaling behavior, i.e. we run the gradient computation on a single instance and vary the number of OpenMP threads. In contrast to the weak scaling experiment, we model wave propagation in the full domain $(1,911 \times 10,789$ grid points), to ensure that the sub-domain of each worker is not too small when we use maximum number of threads. The measured runtime is the sum of the kernel times spent for solving the forward and the adjoint wave equation and therefore excludes memory allocation time and code generation time.

Since AWS Batch runs all jobs as Docker containers, we compare the runtimes with AWS Batch to running our application on a bare metal instance, in which case we have direct access to the compute node and run our code without any virtualization. All timings on AWS are performed on a r5.24xlarge EC2 instance, which is a memory optimized instance type that uses the Intel Xeon Platinum $8175 \mathrm{M}$ architecture. The $24 \mathrm{xlarge}$ instance has 96 virtual CPU cores (48 physical cores on 2 sockets) and $768 \mathrm{~GB}$ of memory. Using the largest possible instance of the $r 5$ class, ensures that our AWS Batch job has exclusive access to the physical compute node. Bare metal instances automatically give users exclusive access to the full node. We also include the Optimum HPC cluster in our comparison, a small research cluster at the University of British Columbia based on the Intel's Ivy Bridge $2.8 \mathrm{GHz}$ E5-2680v2 processor. Optimum has 2 CPUs per node and 10 cores per CPU.

Figure $8 \mathrm{a}$ shows the comparison of the kernel runtimes on AWS and Optimum and Figure $8 \mathrm{~b}$ displays the corresponding speedups. As expected, the $r 5$ bare metal instance shows the best scaling, as it uses a newer architecture than Optimum and does not suffer from the virtualization overhead of Docker. We noticed that AWS Batch in its default mode uses hyperthreading (HT), even if we perform thread pinning and instruct AWS Batch to use separate physical cores. As of now, the only way to prevent AWS Batch from performing HT, is to modify the Amazon Machine Image (AMI) of the corresponding AWS compute environment and set the nr_cpus parameter of the /etc/default/grub file to the number of physical cores per socket (i.e. 24). With HT disabled, the runtimes and speedups of AWS Batch are very close to the timings on the bare-metal instances, indicating that the overhead of Docker affects the runtimes and scaling of our memory-intensive application only marginally, which matches the findings of [73]. 


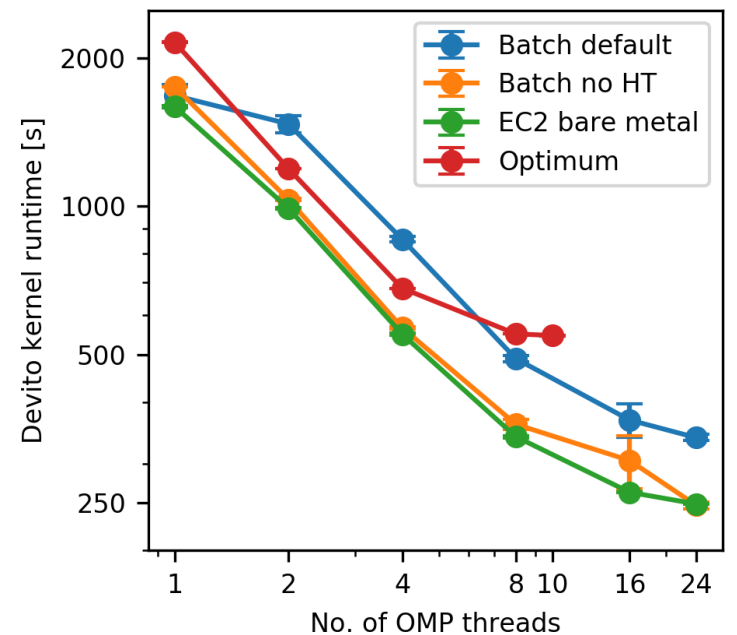

(a)

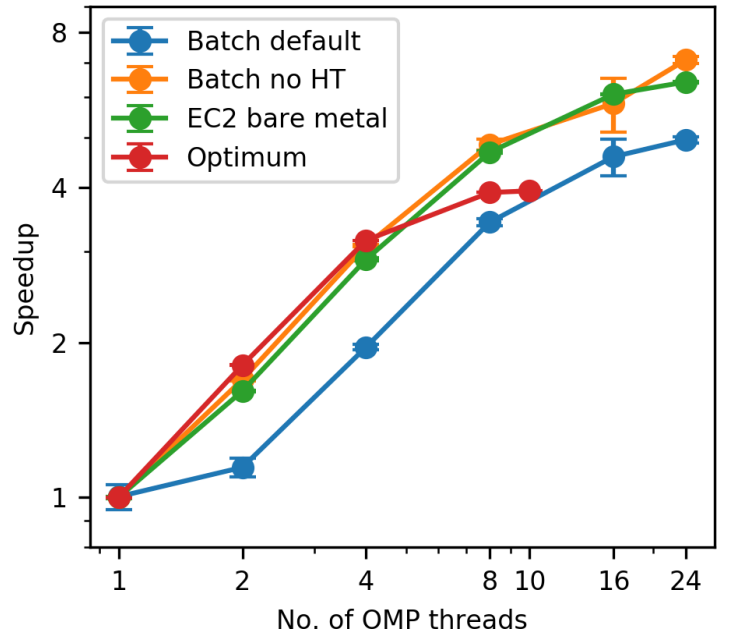

(b)

Figure 8: Strong scaling results for computing a single image gradient of the BP model as a function of the number of threads. Figure (a) shows the runtimes for AWS Batch with and without hyperthreading, as well as the runtimes on the $\mathrm{r} 5$ bare metal instance, in which case no containerization or virtualization is used. For reference, we also provide the runtime on a compute node of an on-premise HPC cluster. Figure (b) shows the corresponding speeds ups.

Next, we analyze the horizontal strong scaling behavior of running our application with AWS Batch. Once again, we consider the computation of one single gradient, but this time we vary the number of EC2 instances on which the underlying wave equations are solved. We would like to emphasize that AWS Batch is used differently than in the weak scaling experiment, where AWS Batch was used to parallelize the sum over source locations. Multiple workloads (i.e. gradients) were submitted to AWS Batch as an array job and communication between workers of an array job is not possible. Here, we submit a single workload (i.e. one gradient) as a multi-node AWS Batch job, in which case IP-based communication between instances is enabled. Since this involves distributed memory parallelism, we use domain decomposition based on message passing (MPI) to solve the wave equations on multiple EC2 instances [74, 75]. The code with the corresponding MPI communication statements is automatically generated by the Devito compiler. Furthermore, we use multi-threading on each individual instance and utilize the maximum number of available cores per socket, which is 24 for the $r 5$ instance and 18 for the $c 5 n$ instance.

We compare the $r 5.24 x$ large instance type from the last section with Amazon's recently introduced c5n HPC instance. Communication between AWS instances is generally based on ethernet and the $r 5$ instances have up to 25 GBps networking performance. The c5n instance type uses Intel Xeon Platinum $8142 \mathrm{M}$ processors with up to $3.4 \mathrm{Ghz}$ architecture and according to AWS provides up to 100 GBps of network bandwidth. The network is based on AWS' Nitro card and the elastic network adapter, but AWS has not disclosed whether this technology is based on Infiniband or Ethernet [76]. Figures $9 \mathrm{a}$ and $9 \mathrm{~b}$ show the kernel runtimes and the corresponding speedups ranging from 1 instance to 16 instances. The r5 instance has overall shorter runtimes than the c5n instance, since the former has 24 physical cores per CPU socket, while the $c 5 n$ instance has 18 . However, as expected, the $c 5 n$ instance type exhibits a better speedup than the $r 5$ instance, due to the better network performance. Overall, the observed speed up on both instances types is excellent, with the $c 5 n$ instance archiving a maximum speedup of 11.3 and the $r 5$ instance of 7.2.

The timings given in Figure $9 \mathrm{a}$ are once again the pure kernel times for solving the PDEs, but a breakdown of the components that make up the total time-to-solution on the $\mathrm{c} 5 \mathrm{n}$ instance is provided in Figure 9c The job runtime is defined as the interval between the time stamp at which the batch job was created and the S3 time stamp of the computed gradient. As in our weak scaling test, this includes the time for AWS Batch to request and launch the EC2 instances and to start the Docker containers, but excludes the gradient summation time, since we are only considering the computation of a single gradient. The container runtime is the runtime of the Docker container on the master node and includes the time it takes AWS Batch to launch the remaining workers and to establish an ssh connection between all instances/containers. Currently, AWS Batch requires this process to be managed by the user using a shell script that is run inside each container. After a connection to all workers has been established, the containers run the application as a Python program on each worker. The Python runtime in Figure $9 \mathrm{c}$ is defined as the runtime of Python on the main node and includes reading the seismic data from S3, allocating memory and Devito's code generation. Our timings in Figure 9c show that the overhead from requesting instances and establishing a cluster, i.e. the difference between the Python and container runtime, is reasonable for a small number of instances (less than 2 minutes), but grows significantly as the number instances is increased to 8 and 16. Depending on the runtime of the application, the overhead thus takes up a significant amount of the 


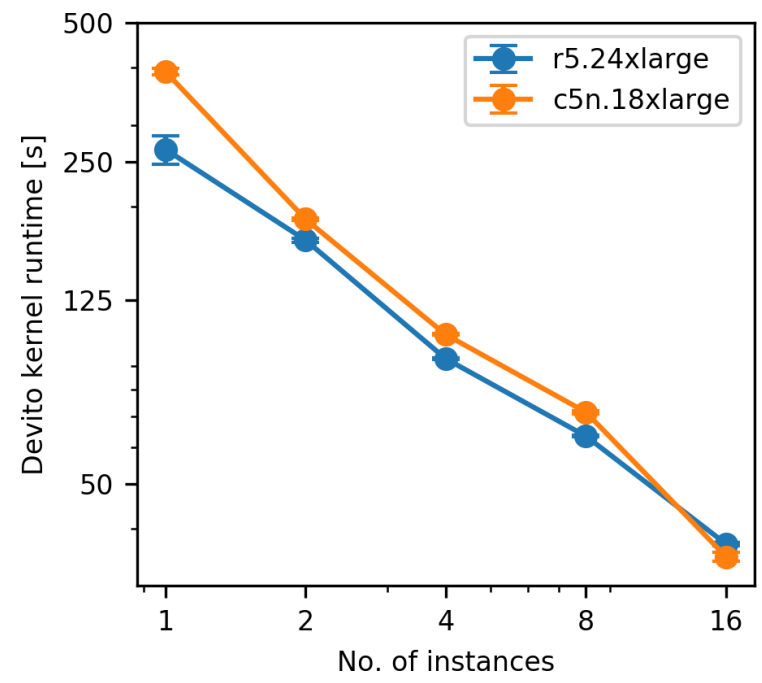

(a)

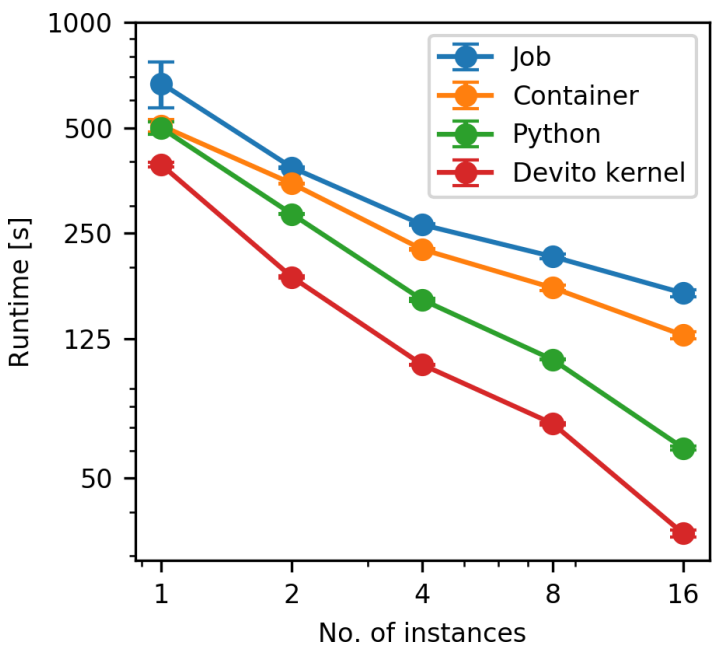

(c)

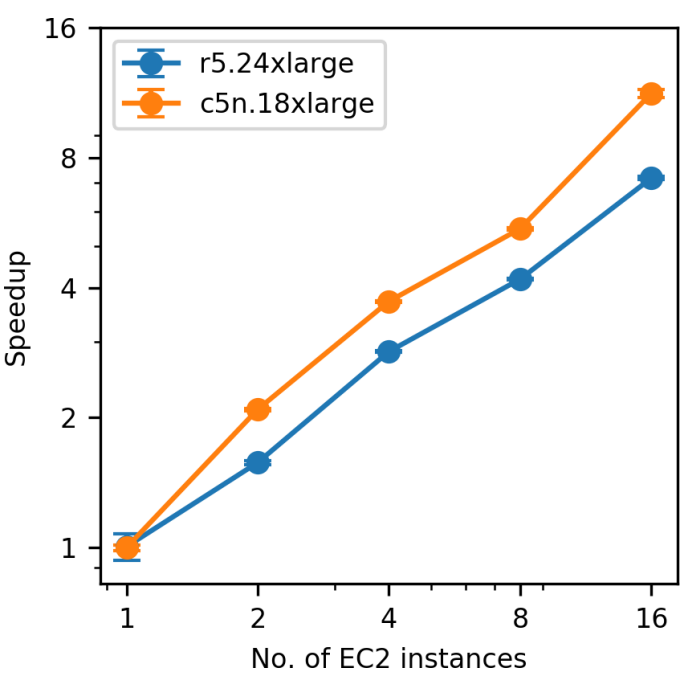

(b)

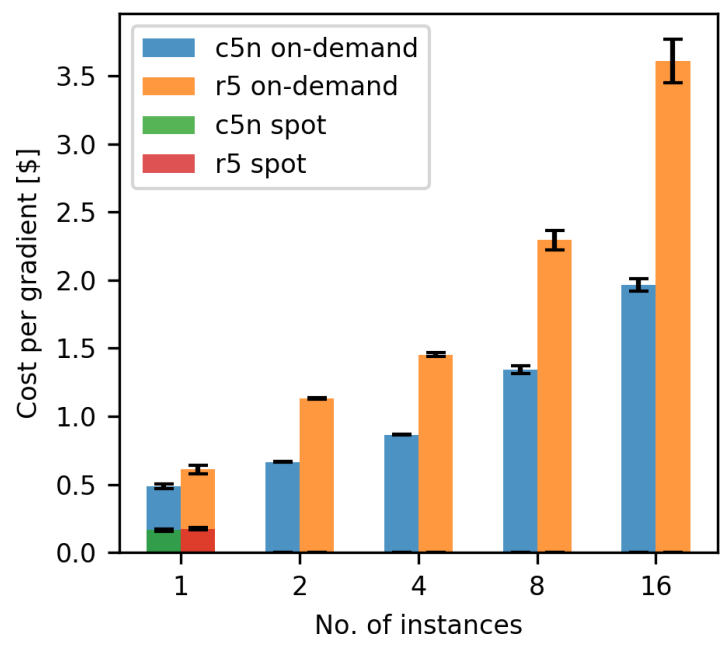

(d)

Figure 9: Strong scaling results for computing a single gradient as an AWS Batch multi-node job for an increasing number of instances. Figures (a) and (b) show the Devito kernel times and speedups on two different instance types. The observed speedups are 11.3 for the $c 5 n$ and 7.2 for the $r 5$ instance. Figure (c) shows a breakdown of the time-to-solution of each batch job into its individual components. Figure (d) shows the EC2 cost for computing the gradients. The spot price is only provided for the single-instance batch jobs, as spot instances are not supported for multi-node batch jobs.

time-to-solution. In our example, this was the case for 8 and 16 instances, but for more compute-heavy applications that run for one or multiple hours, this amount of overhead may still be acceptable.

Figure $9 \mathrm{~d}$ shows the cost for running our scaling test as a function of the cluster size. The cost is calculated as the instance price (per second) times the runtime of the container on the main node times the number of instances. The cost per gradient grows significantly with the number of instances, as the overhead from establishing an ssh connection to all workers increases with the cluster size. The communication overhead during domain decomposition adds an additional layer of overhead that further increases the cost for an increasing number of instances. This is an important consideration for HPC in the cloud, as the shortest time-to-solution does not necessarily correspond to the cheapest approach. Another important aspect is that AWS Batch multi-node jobs do not support spot instances [75]. Spot instances allow users to access unused EC2 capacities at significantly lower price than at the on-demand price, but AWS can terminate spot instances at any time with a two minute warning, e.g. if the demand for that instance type increases [52]. Spot instances are typically in the range of 2 to 3 times cheaper than the corresponding on-demand price, but AWS Batch multi-node jobs are, for the time being, only supported with on-demand instances. 


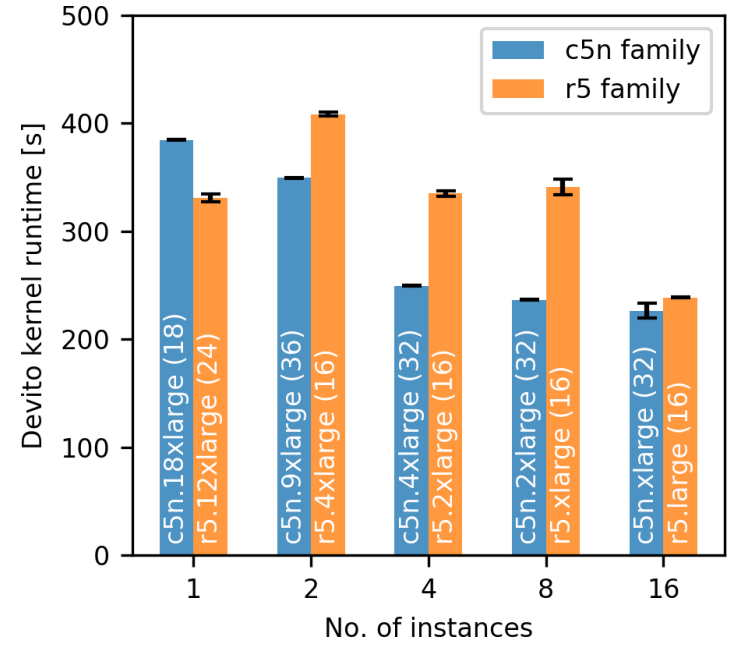

(a)

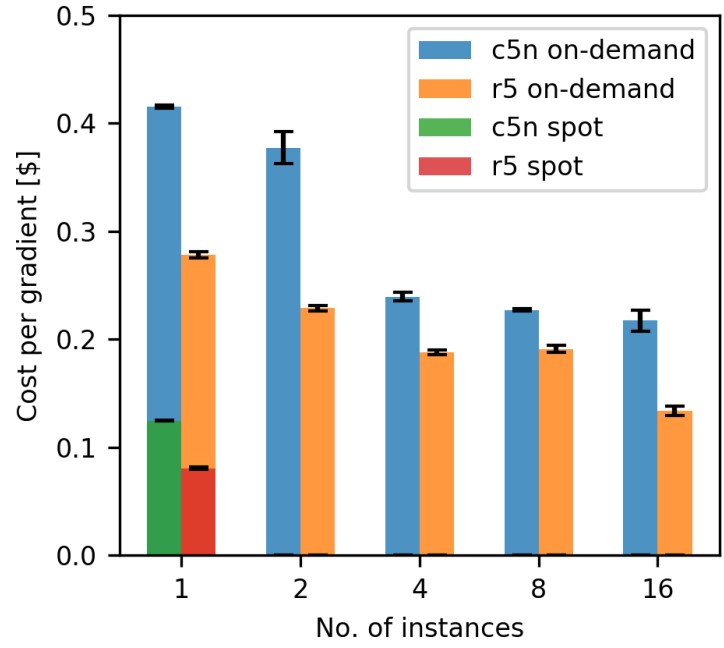

(b)

Figure 10: Devito kernel runtimes for computing a single gradient as an AWS Batch job for an increasing number of instances. In comparison to the previous example, we use the smallest possible instance type for each job, as specified in each bar. We use the maximum number of available cores on every instance type and the total number of cores across all instances is given in each bar. Figure (b) shows the corresponding cost for computing the gradients. Choosing the instance size such the total memory is approximately constant, avoids that the cost increases as a larger number of instances are used per gradient. However, single instances using spot prices ultimately remain the cheapest option.

The scaling and cost analysis in Figures $9 \mathrm{a}-9 \mathrm{~d}$ was carried out on the largest instances of the respective instance types ( $5.24 \mathrm{xlarge}$ and $\mathrm{c5n} .18 \mathrm{xlarge}$ ) to guarantee exclusive access to the compute nodes and network bandwidth. Increasing the number of instances per run therefore not only increases the total number of available cores, but also the amount of memory. However, for computing a single gradient, the required amount of memory is fixed, so increasing the number of instances reduces the required amount of memory per instance, as wavefields are distributed among more workers. In practice, it therefore makes sense to chose the instance type based on the required amount of memory per worker, as memory is generally more expensive than compute. In our specific case, computing the gradient requires $170 \mathrm{~GB}$ of memory, which requires either a single $\mathrm{r} 5.12 \mathrm{xl}$ arge instance, two $\mathrm{r} 5.4 \mathrm{xlarge}$, four $\mathrm{r} 5.2 \mathrm{xlarge}$, eight $r 5 . x l a r g e$ or sixteen $r 5$. large instances. However, these instances not only differ in the amount of memory, but also in the number of CPU cores. We repeat our previous scaling test, but rather than using the same instance type in all runs, we choose the instance type based on the required amount of memory. Furthermore, for every instance type, we utilize the maximum amount of available cores using multi-threading with OpenMP. The kernel runtimes for an increasing number of instances is shown in Figure 10a. In each bar, we indicate which instance type was used, as well as the total number of cores across all instances. The corresponding costs for computing each gradient is shown in Figure 10a Compared to the previous example, we observe that using 16 small on-demand instances leads to a lower cost than using a single more expensive large instance, but that using a single instance ultimately remains the most cost-effective way of computing a gradient, due to the possibility to utilize spot instances.

Table 1: Comparison of parallelization strategies on a single EC2 instance in the context of AWS Batch. The timings are the Devito kernel times for computing a single gradient of the BP model using AWS Batch. The program runs as a single docker container on an individual EC2 instance, using either multi-threading (OpenMP) or a hybrid approach of multithreading and domain-decomposition (OpenMP + MPI).

\begin{tabular}{llll}
\hline Grid & CPU (cores) & Parallelization & Runtime [s] \\
\hline $1,911 \times 5,394$ & $1(24)$ & OMP & $190.17 \pm 7.12$ \\
$1,911 \times 10,789$ & $1(24)$ & OMP & $378.94 \pm 13.57$ \\
$1,911 \times 10,789$ & $2(48)$ & OMP & $315.92 \pm 16.50$ \\
$1,911 \times 10,789$ & $2(48)$ & OMP + MPI & $249.13 \pm 5.22$ \\
\hline
\end{tabular}

In terms of cost, our scaling examples underline the importance of choosing the EC2 instances for the AWS Batch jobs based on the total amount of required memory, rather than based on the amount of CPU cores. Scaling horizontally by using 
an increasingly large number of instances expectedly leads to a faster time-to-solution, but results in a significant increase of cost as well (Figure 9d). As shown in Figure 10b this increase in cost can be avoided to some extent by choosing the instance size such that the total amount of memory stays approximately constant, but ultimately the restriction of not supporting spot instances, makes multi-node batch jobs not attractive in scenarios where single instances provide sufficient memory to run a given application. In practice, it makes therefore sense to use single node/instance batch jobs and to utilize the full number of available cores on each instance. The largest EC2 instances of each type (e.g. r5.24xlarge, c5n.18xlarge) have two CPU sockets with shared memory, making it possible to run parallel programs using either pure multi-threading or a hybrid MPI-OpenMP approach. In the latter case, programs still run as a single Docker container, but within each container use MPI for communication between CPU sockets, while OpenMP is used for multithreading on each CPU. For our example, we found that computing a single gradient of the BP model with the hybrid MPI-OpenMP approach leads to a $20 \%$ speedup over the pure OpenMP program (Table 1 , which correspondingly leads to $20 \%$ cost savings as well.

\subsection{Cost comparison}

One of the most important considerations of high performance computing in the cloud is the aspect of cost. As users are billed for running EC2 instances by the second, it is important to use instances efficiently and to avoid idle resources. This is oftentimes challenging when running jobs on a conventional cluster. In our specific application, gradients for different seismic source locations are computed by a pool of parallel workers, but as discussed earlier, computations do not necessarily complete at the same time. On a conventional cluster, programs with a MapReduce structure, such as parallel gradient computations, are implemented based on a client-server model, in which the workers (i.e. the clients) compute the gradients in parallel, while the master (the server) collects and sums the results. This means that the process has to wait until all gradients $\mathbf{g}_{i}$ have been computed, before the gradient can be summed and used to update the image. This inevitably causes workers that finish their computations earlier than others to the sit idle. This is problematic when using a cluster of EC2 instances, where the number of instances are fixed, as users have to pay for idle resources. In contrast, the event-driven approach based on Lambda functions and AWS Batch automatically terminates EC2 instances of workers that have completed their gradient calculation, thus preventing resources from sitting idle. This fundamentally shifts the responsibility of requesting and managing the underlying EC2 instances from the user to the cloud environment and leads to significant cost savings as demonstrated in the following example.

We illustrate the difference between the event-driven approach and using a fixed cluster of EC2 instances by means of a specific example. We consider our previous example of the BP synthetic model and assume that we want to compute the gradient for a batch size of 100. As in our weak scaling experiment, we restrict the modeling domain to the subset of the model that includes the respective seismic source location, as well as the seismic receivers that record the data. Towards the edge of the model, the modeling domain is smaller, as some receivers lie outside the modeling domain and are therefore omitted. We compute the gradient $\mathbf{g}_{i}$ for 100 random source locations and record the runtimes (Figure 11a). We note that most gradients take around 250 seconds to compute, but that the runtimes vary due to different domain sizes and varying EC2 capacity (similar to the timings in Figure 6c). We now model the idle times for computing these gradients on a cluster of EC2 instances as a function of the the number of parallel instances, ranging from 1 instance (fully serial) to 100 instances (fully parallel). For a cluster consisting of a single instance, the cumulative idle time is naturally zero, as the full workload is executed in serial by a single instance. For more than one instance, we model the amount of time that each instance is utilized, assuming that the workloads are assigned dynamically to the available instances. The cumulative idle time $t_{\text {idle }}$ is then given as the sum of the differences between the runtime of each individual instance $t_{i}$ and the instance with the longest runtime:

$$
t_{\mathrm{idle}}=\sum_{i=1}^{n_{\mathrm{EC} 2}}\left(\max \left\{t_{i}\right\}-t_{i}\right),
$$

The cumulative idle time as a function of the cluster size $n_{\mathrm{EC} 2}$ is plotted in Figure $11 \mathrm{~b}$. We note that the cumulative idle time generally increases with the cluster size, as a larger number of instances sit idle while waiting for the final gradient to be computed. On a cluster with 100 instances each gradient is computed by a separate instance, but all workers have to wait until the last worker finishes its computation (after approximately 387 seconds). In this case, the varying time-to-solutions of the individual gradients leads to a cumulative idle time of 248 minutes. Compared to the cumulative computation time of all gradients, which is 397 minutes, this introduces an overhead of more than 60 percent, if the gradients are computed on a cluster with 100 instances. The cumulative idle time is directly proportional to the cost for computing the 100 gradients, which is plotted on the right axis of Figure $11 \mathrm{~b}$ With AWS Batch, the cumulative idle time for computing the 100 gradients is zero, regardless of the number of parallel instances that AWS Batch has access to. Any EC2 instance that is not utilized anymore is automatically shut down by AWS Batch, so no additional cost other than the pure computation time of the gradients is invoked [77].

In practice, it is to be expected that the cost savings of AWS Batch are even greater, as we are not taking the time into account that it takes to start an EC2 cluster of a specified number of instances. In our weak scaling experiments (Figure 6a), we found that spinning up a large number of EC2 instances does not happen instantaneously but over a period of several minutes, so starting a cluster of EC2 instances inevitably causes some instances to sit idle while the remaining instances are started. This was also observed for our multi-node AWS Batch job experiment (Figure 9c), but in this case the cluster size per gradient is considerably smaller than the size of a single large cluster for computing all gradients. Single-node AWS 


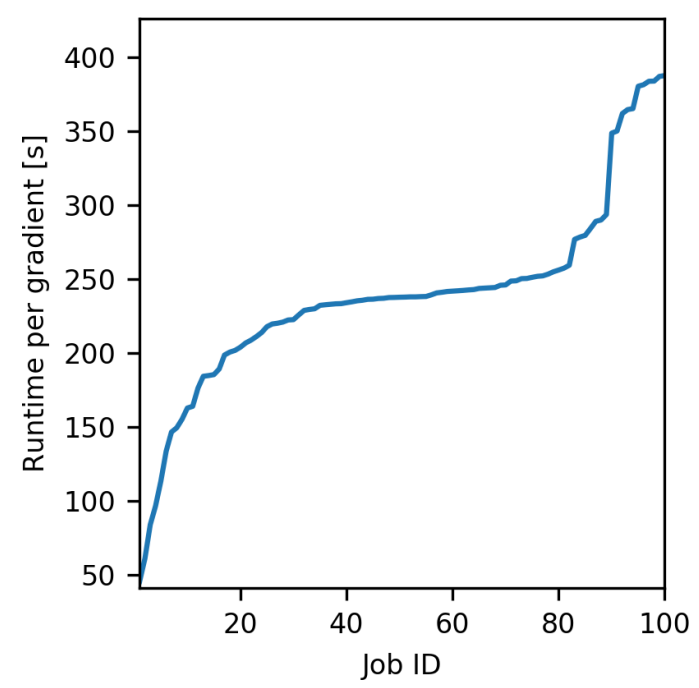

(a)

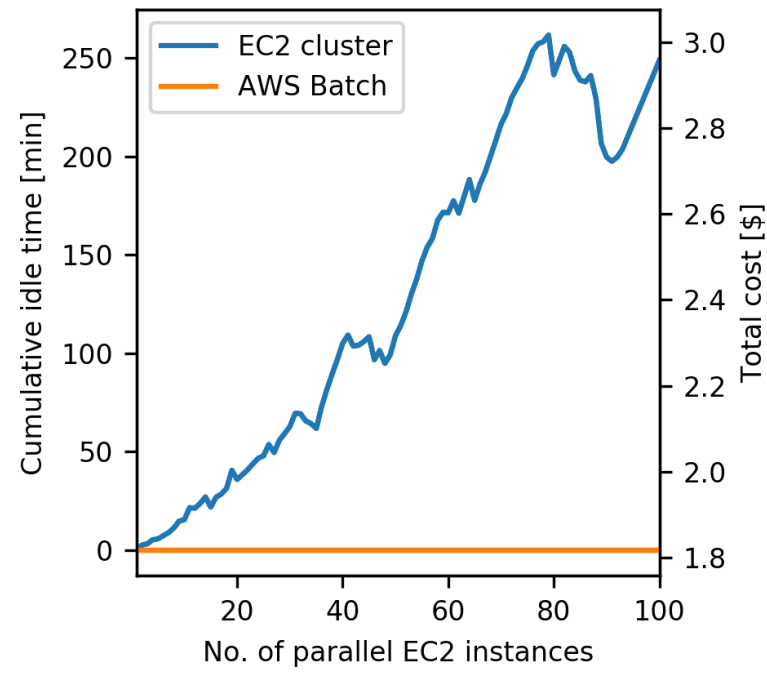

(b)

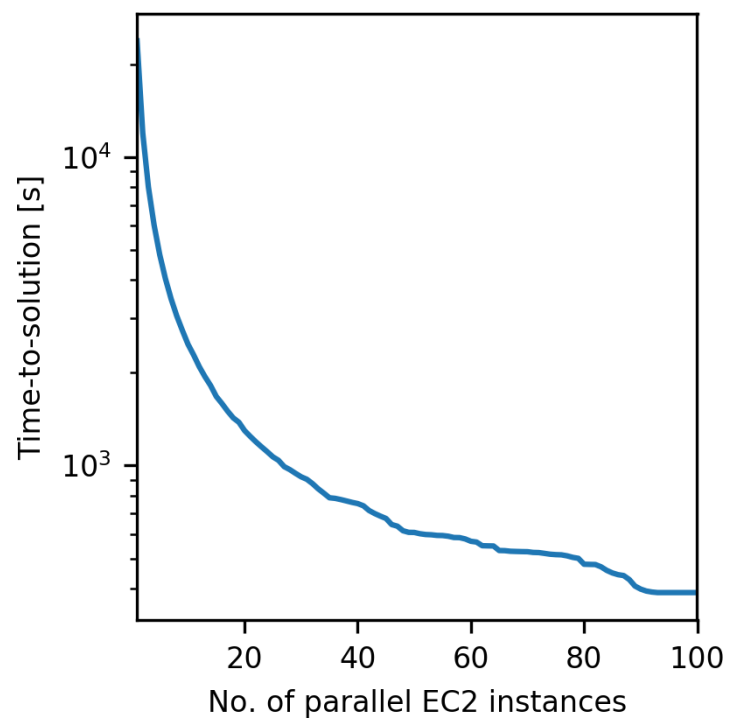

(c)

Figure 11: (a) Sorted container runtimes of an AWS Batch job in which we compute the gradient of the BP model for a batch size of 100. Figure (b) shows the cumulative idle time for computing this workload as a function of the number of parallel workers on either a fixed cluster of EC2 instances or using AWS Batch. The right-hand y-axis shows the corresponding cost, which is proportional to the idle time. In the optimal case, i.e. no instances every sit idle, the cost for computing a gradient of batch size 100 is $1.8 \$$. Figure (c) shows the time-to-solution as a function of the number of parallel instances, which is the same on an EC2 cluster and for AWS Batch, if we ignore the startup time of the AWS Batch workers or of the corresponding EC2 cluster.

Batch jobs do not suffer from the variable startup time, as workers that are launched earlier than others instantaneously start their computations, without having to wait for the other instances.

While computing the 100 gradients on an EC2 cluster with a small number of instances results in little cumulative idle time, it increases the overall time-to-solution, as a larger number of gradients have to be sequentially computed on each instance (11c). With AWS Batch this trade-off does not exist, as the cumulative idle time, and therefore the cost for computing a fixed workload, does not depend on the number of instances. However, it is to be expected that in practice the time-to-solution is somewhat larger for AWS Batch than for a fixed cluster of EC2 instances, as AWS Batch needs to request and launch EC2 instances for every new gradient computation. In our weak scaling experiments in which we requested up to 128 instances, we found that the corresponding overhead lies in the range of 3 to 10 minutes (per iteration), 
but it is to be expected that the overhead further grows for an even larger number of instances. However, no additional cost is introduced while AWS Batch waits for the EC2 instances to start.

\subsection{Cost saving strategies for AWS Batch}

Using AWS Batch for computing the gradients of a seismic imaging workflow limits the runtime that each EC2 instance is active to the amount of time it takes to compute a single gradient (of one source location). Running a seismic imaging workflow on a conventional cluster of EC2 instances, requires instances to stay up during the entire execution time of the program, i.e. for all iterations of the seismic imaging optimization algorithm. The limitation of instance runtimes to the duration of a single gradient computations with AWS Batch is beneficial for the usage of spot instances, as it reduces the chance that a specific instance is shut down within the duration it is used. As demonstrated in our earlier examples (Figures 9d and 10b), spot instances can significantly reduce the cost of running EC2 instances, oftentimes by a factor of 2-4 in comparison to on-demand instances (Table 2,.

Table 2: AWS on-demand and spot prices of a selection of EC2 instance types that were used in the previous experiments. Prices are provided for the US East (North Virginia) region, in which all experiments were carried out $(07 / 11 / 2019)$.

\begin{tabular}{llll}
\hline Instance & On-demand (\$/hour) & Spot (\$/hour) & Ratio \\
\hline m4.4xlarge & 0.800 & 0.2821 & 2.84 \\
r5.24xlarge & 6.048 & 1.7103 & 3.54 \\
c5n.18xlarge & 3.888 & 1.1659 & 3.33 \\
\hline
\end{tabular}

In contrast to on-demand instances, the price of spot instances is not fixed and depends on the current demand and availability of the instance type that is being requested. As such, prices for spot instances can vary significantly between the different zones of a specific region (Figure 12a). If spot instances are used for a cluster and are fixed for the entire duration of the program execution, users are exposed to variations of the spot price during that time period. This effects is usually negligible for programs that run in a matter of hours, as spot prices typically do not vary substantially over short periods of time. However, large-scale 3D seismic imaging problems potentially run over the course of multiple days, in which case varying spot prices can have significant influence on the cost.

We consider a hypothetical example in which we assume that we run a large-scale imaging example for 20 iterations of an optimization algorithm, where each iteration takes 12 hours to compute, which leads to a total runtime of 10 days. Figures 12a shows the historical spot price of the c5n.18xlarge instance over a 10 day time period in April 2019 and Figure $12 \mathrm{~b}$ shows the normalized cost of running the example in each specific zone. We note that the spot price of this instance type is the same across all zones at the start of the program, but that the spot price in the us-east-1b zone starts increasing significantly after a few hours. In this case, running the example on a fixed cluster of instances results in cost differences of 40 percent, depending on which zone was chosen at the start of the program. Our event-driven workflow with AWS Batch, allows that the cheapest available zone is automatically chosen at the start of every iteration, which ensures that zones that exhibit a sudden increase of the spot price, such as zone us-east-1b, are avoided in the subsequent iteration.

Another example for a different time interval and the $c 1 . x$ large instance type is shown in Figure $12 \mathrm{c}$ and the relative cost of running the example in the respective zone is plotted in Figure 12d The right-most bar shows the cost if the example was run with AWS Batch and the event-driven workflow had chosen the cheapest available zone at each iteration. In this case, switching zones between iterations leads to cost savings of 15 percent in comparison to running the example in the us-east-1a zone, which is the cheapest zone at the start of the program. For our cost estimates (Figure 12b and 12d), we assume that the spot price is not affected by our own application, i.e. by our own request of a potentially large number of spot instances, but in practice this issue needs to be taken into account as well. Overall, the two examples shown here are obviously extreme cases of price variations across zones and in practice the spot price is oftentimes reasonably stable. However, spot price fluctuations are nevertheless unpredictable and the event-driven approach with AWS Batch allows to minimize exposure to price variations.

Apart from choosing spot instances in different zones, it is also possible to vary the instance type in that is used for the computations. For example, in Figure 13a we plot the historical spot price of the xlarge instance for various instance types (m5, c5, c5n and r5). All instances have 4 virtual CPUs, but vary in the amount of memory and their respective CPU architecture. Spot instances are not priced proportionally to their hardware (memory, cores, architecture), but based on the current demand. Therefore, it is oftentimes beneficial to compare different instance types and choose the currently cheapest type from a pool of possible instances. As before, we compare the relative cost for running the 10 day example on a cluster of EC2 instances, in which case the instance type is fixed for the duration of the program, against the dynamic approach with AWS Batch. Again, the event-driven approach allows to minimize exposure to price changes over the duration of the example, by choosing the cheapest available instance type at the beginning of each iteration. 


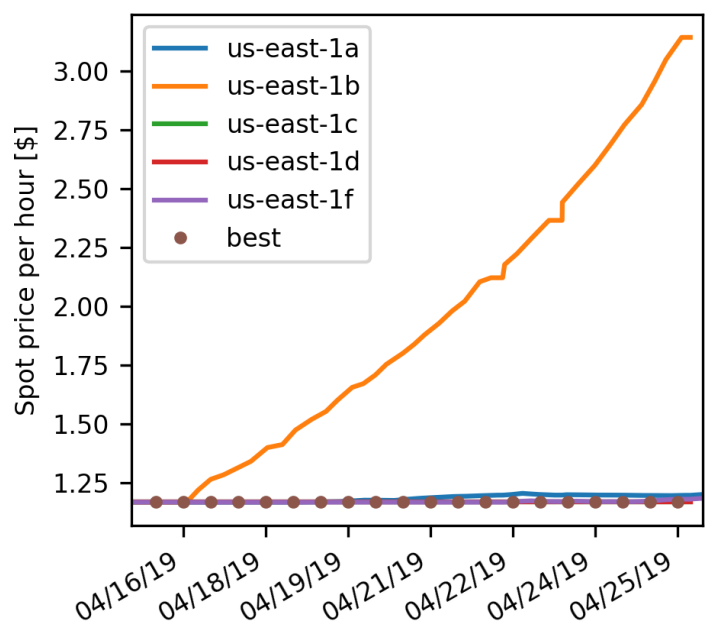

(a)

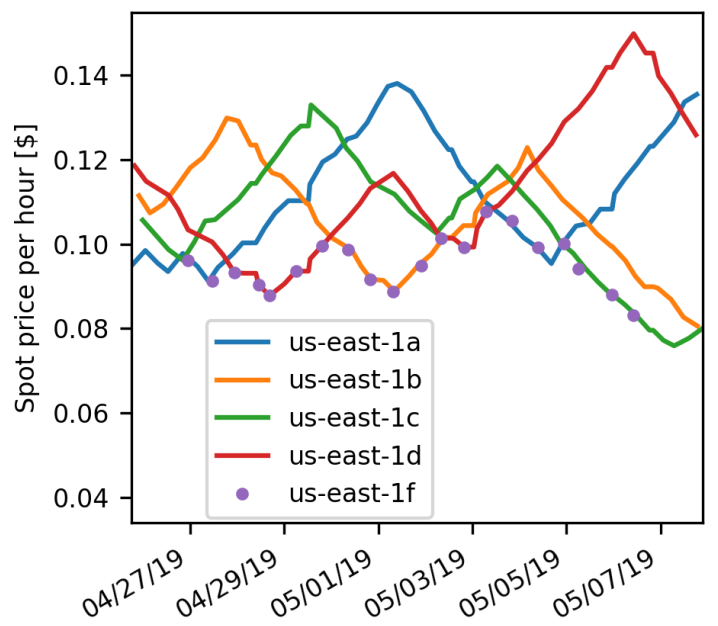

(c)

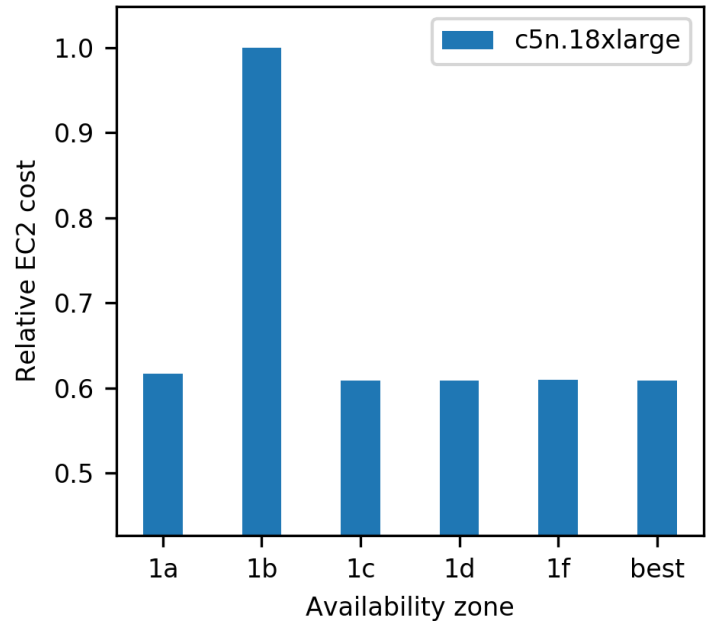

(b)

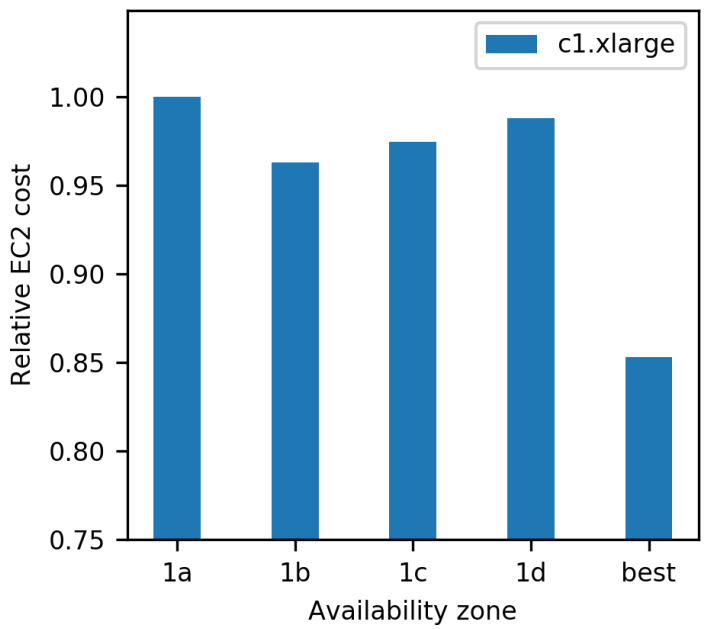

(d)

Figure 12: (a) Historical spot price of the c5n.18xlarge instance in different zones of the US East region over a 10 ten day period in April 2019. Figure (b) shows the relative cost for running an iterative seismic imaging algorithm over this time period in the respective zones. The right-most bar indicates the price for running the application with our event-driven workflow, in which the cheapest zone is automatically chosen at the start of each iteration (indicated as dots in Figure a). Figures (c) and (d) are the same plots for the c1.xlarge instance during a different time window.

\subsection{Resilience}

In the final experiment of our performance analysis, we analyze the resilience of our workflow and draw a comparison to running an MPI program on a conventional cluster of EC2 instances. Resilience is an important factor in high performance computing, especially for applications like seismic imaging, whose runtime can range from several hours to multiple days. In the cloud, the mean-time-between failures is typically much shorter than on comparable HPC systems [9], making resilience potentially a prohibiting factor. Furthermore, using spot instances further increases the exposure to instance shut downs, as spot instances can be terminated at any point in time with a two minute warning.

Seismic imaging codes that run on conventional HPC clusters typically use MPI to parallelize the sum of the source indices. MPI based applications exhibit a well known shortcoming of having a relatively low fault tolerance, as hardware failures lead to the termination of a running program. Currently, the only noteworthy approach of fault tolerance for MPI programs is the User Level Fault Mitigation (ULFM) Standard [78]. ULFM enables an MPI program to continue running after a node/instance failure, using the remaining available resources to finish the program execution. Using AWS Batch to compute the gradients $\mathbf{g}_{i}$ for a given batch size, provides a natural fault tolerance, as each gradient is computed by 


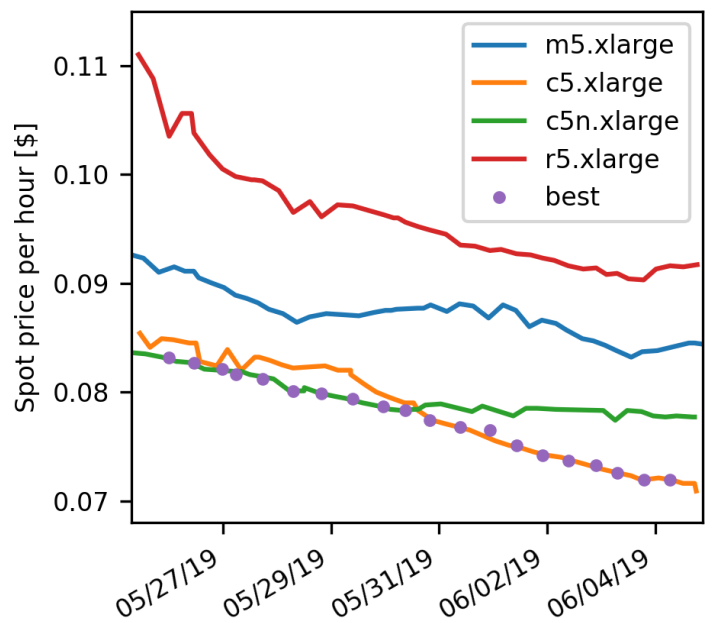

(a)

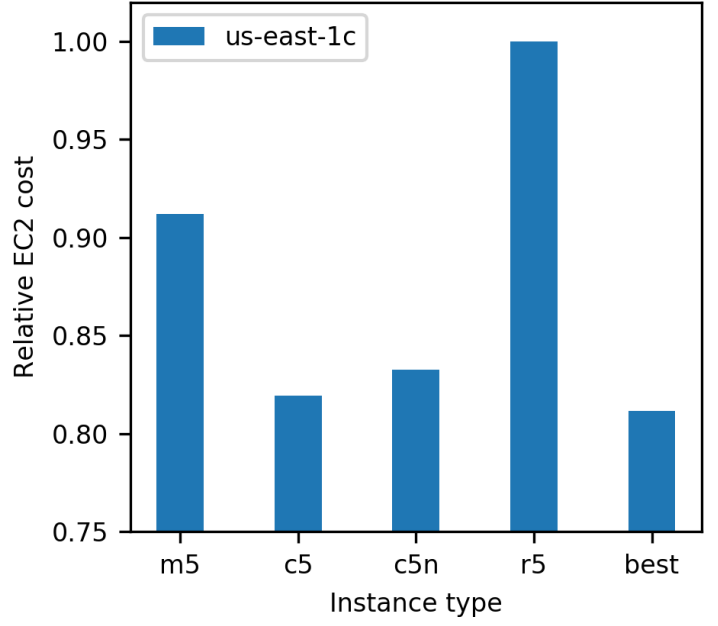

(b)

Figure 13: (a) Historical spot prices for a variety of 4xlarge instances over a 10 day period in April 2019. All shown instances have $128 \mathrm{~GB}$ of memory, but vary in their number of CPU cores and architectures. Figure (b) shows the relative cost of running an iterative seismic imaging application over this time period in the respective zone and for the case, in which the cheapest available instance is chosen at the beginning of each iteration.

a separate container, so the crash of one instance does not affect the execution of the code on the remaining workers. Furthermore, AWS Batch provides the possibility to automatically restart EC2 instances that have crashed. In contrast to ULFM, this allows the completion of programs with the initial number of nodes or EC2 instances, rather than with a reduced number.

We illustrate the effect of instance restarts by means of our previous example with the BP model (Figure 5). Once again, we compute the gradient of the LS-RTM objective function for a batch size of 100 and record the runtimes without any instance/node failures. We compute the gradients using two different computational strategies for backpropagation. In the first approach, we compute the gradient on instances with a sufficient amount of memory to store the state variables in memory, which leads to an average runtime per gradient of 5 minutes (using 62 GB of memory). In the second approach, we compute the gradients on instances with less memory and use optimal checkpointing [43], in which case we store only a small subset of state variables and recompute the remaining states during backpropagation. This increases the average runtime per gradient to 45 minutes, but also reduces the required amount of memory for this example from 62 GB to 5 GB.

We then model the time that it takes to compute the 100 gradients for an increasing number of instance failures with and without restarts. We assume that the gradients are computed fully in parallel, i.e. on 100 parallel instances and invoke an increasing number of instance failures at randomly chosen times during the execution of program. Without instance restarts, we assign the workload of the failed instances to the workers of the remaining instances and model how long it takes complete the computation of the 100 gradients. With restarts, we add a two minute penalty to the failed workers and then restart the computation on the same instance. The two minute penalty represents the average amount of time it takes AWS to restart a terminated EC2 instance and was determined experimentally by manually shutting down EC2 instances of an AWS Batch job and recording the time it takes to restart the container on a new instance.

Figure 14 shows the ratio of the time-to-solution for computing the 100 gradients without events (i.e. without failures) to the modeled time-to-solution with events. This ratio is known as the resilience factor [79] and provides a metric of how instance failures affect the time-to-solution and therefore the cost of running a given application in the cloud:

$$
r=\frac{\text { time-to-solution event-free }}{\text { time-to-solution event }}
$$

Ideally, we aim for this factor being as close to 1 as possible, meaning that instance failures do not significantly increase the time-to-solution. Figures $14 \mathrm{a}$ and $14 \mathrm{~b}$ show the resilience factors with and without restarts for the two different backpropagation strategies, which represent programs of different runtimes. The resilience factor is plotted as a function of the percentage of instance failures and is the average of 10 realizations, with the standard deviation being depicted by the shaded colors. The plots show that the largest benefit from being able to restart instances with AWS Batch is achieved for long running applications (Figure 14b). The resilience factor with instance restarts approaches a value of 0.5 , since in the worst case, the time-to-solution is doubled if an instance fails shortly before completing its gradient computation. Without being able to restart instances, as would be the case for MPI programs with ULFM, the gradient computations need to be completed by the remaining workers, so the resilience factor continuously decreases as the failure percentage 


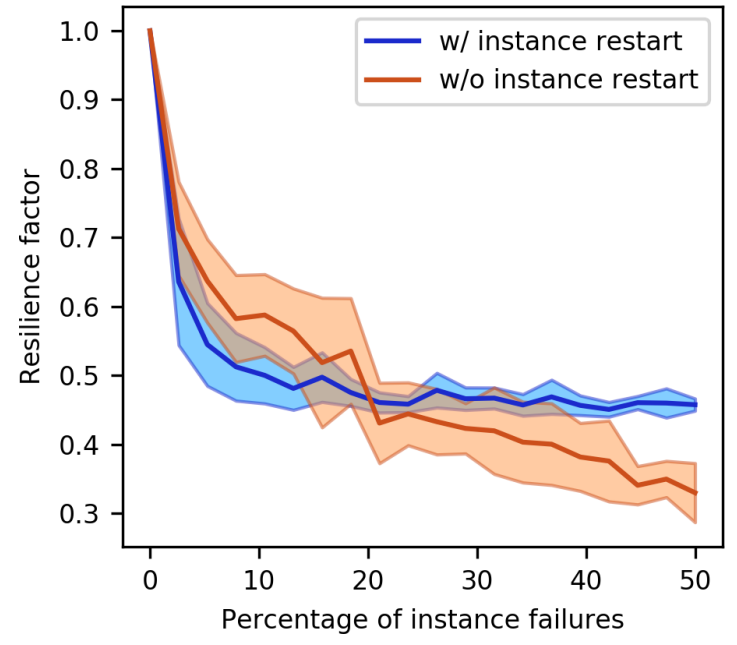

(a)

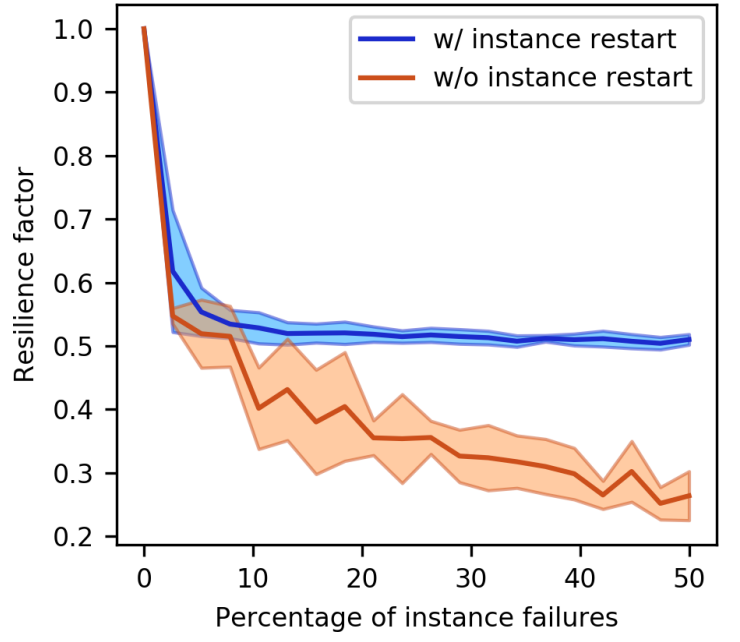

(b)

Figure 14: Comparison of the resilience factor (RF) for an increasing percentage of instance failures with and without instance restarts. The RF provides the ratio between the original runtime for computing the gradient of the BP model for a batch size of 100 and the runtime in the presence of instance failures. Figure (a) is the $\mathrm{RF}$ for an application that runs for 5 minutes without failures, while figure (b) is based on an example whose original time-to-solution is 45 minutes.

increases. For short running applications (Figure 14a), the overhead of restarting instances diminishes the advantage of instance restarts, unless a significant percentage of instances fail, which, however, is unlikely for programs that run in a matter of minutes. On the other hand, long running programs or applications with a large number of workers are much more likely to encounter instance shut downs and our experiment shows that these programs benefit from the automatic instance restarts of AWS Batch.

\section{Discussion}

The main advantage of an event-driven approach based on AWS Batch and Lambda functions for seismic imaging in the cloud is the automated management of computational resources by AWS. EC2 instances that are used for carrying out heavy computations, namely for solving large-scale wave equations, are started automatically in response to events, which in our case are Step Functions advancing the serverless workflow to the ComputeGradients state. Expensive EC2 instances are thus only active for the duration it takes to compute one element $\mathbf{g}_{i}$ of the full or mini-batch gradient and they are automatically terminated afterwards. Summing the gradients and updating the variables (i.e. the seismic image) is performed on cheaper Lambda functions, with billing being again solely based on the execution time of the respective code and the utilized memory. The cost overhead introduced by Step Functions, SQS messages and AWS Batch is negligible compared to the cost of the EC2 instances that are required for the gradient computations, while cost savings from spot instances and eliminating idle EC2 instances lead to significant cost savings, as shown in our examples. With the benefits of spot instances (factor 2-3), avoidance of idle instances and the overhead of spinning clusters (factor 1.5-2), as well as improved resilience, we estimate that our event-driven workflow provides cost savings of up to an order of magnitude in comparison to using fixed clusters of (on-demand) EC2 instances.

The second major advantage of the proposed approach is the handling of resilience. Instead of running as a single program, our workflow is broken up into its individual components and expressed through Step Function states. Parallel programs based on MPI rely on not being interrupted by hardware failures during the entire runtimetime of the code, making this approach susceptible to resilience issues. Breaking a seismic imaging workflow into its individual components, with each component running as an individual (sub-) program and AWS managing their interactions, makes the event-driven approach inherently more resilient. On the one hand, the runtime of individual components, such as computing a gradient or summing two arrrays, is much shorter than the runtime of the full program, which minimizes the exposure to instance failures. On the other hand, AWS provides built-in resilience for all services used in the workflow. Exceptions in AWS Batch or Lambda lead to computations being restarted, while Step Functions allow users to explicitly define their behavior in case of exceptions, including the re-execution of states. Similarly, messages in an SQS queue have guaranteed at-least-once delivery, thus preventing messages from being lost. Finally, computing an embarrassingly parallel workload with AWS Batch, rather than as a MPI-program, provides an additional layer of resilience, as AWS Batch processes each item from its 
Table 3: An overview how the AWS services used in our workflow map to other cloud providers.

\begin{tabular}{lll}
\hline Amazon Web Services & Microsoft Azure & Google Cloud \\
\hline Elastic Compute Cloud & Virtual Machines & Compute Engine \\
Simple Storage System & Blob storage & Cloud Storage \\
AWS Batch & Azure Batch & Pipelines \\
Lambda Functions & Azure Functions & Cloud Functions \\
Step Functions & Logic Apps & N/A \\
Simple Message Queue & Queue Storage & Cloud Pub/Sub \\
Elastic File System & Azure Files & Cloud Filestore \\
\hline
\end{tabular}

queue separately on an individual EC2 instance and Docker container. Instance failures therefore only affect the respective gradient and computations are automatically restarted by AWS Batch.

The most prominent disadvantage of the event-driven workflow is that EC2 instances have to be restarted by AWS Batch in every iteration of the workflow. In our performance analysis, we found that the overhead of requesting EC2 instances and starting the Docker container lies in the range of several minutes and depends on the overall batch size and on how many instances are requested per gradient. The more items are submitted to a batch queue, the longer it typically takes AWS Batch to launch the final number of instances and to process all items from the queue in parallel. On the other hand, items that remain momentarily in the batch queue, do not incur any cost until the respective EC2 instance is launched. The overhead introduced by AWS Batch therefore only increases the time-to-solution, but does not affect the cost negatively. Due to the overhead of starting EC2 instances for individual computations, our proposed workflow is therefore applicable if the respective computations (e.g. computing gradients) are both embarrassingly parallel and take a long time to compute; ideally in the range of hours rather than minutes. We therefore expect that the advantages of our workflow will be even more prominent when applied to 3D seismic data sets, where computations are orders of magnitude more expensive than in 2D. Devito provides a large amount of flexibility regarding data and model parallelism and allows us to address the large memory imprint of backpropagation through techniques like optimal checkpointing or on-the-fly Fourier transforms, thereby presenting all necessary ingredients to apply our workflow to large-scale 3D models.

Our application, as expressed through AWS Step Functions, represents the structure of a generic gradient-based optimization algorithm and is therefore applicable to problems other than seismic imaging and full-waveform inversion. The design of our workflow lends itself to problems that exhibit a certain MapReduce structure, namely they consists of a computationally expensive, but embarrassingly parallel Map part, as well as a computationally cheaper to compute Reduce part. On the other hand, applications that rely on dense communications between workers or where the quantities of interest such as gradients or functions values are cheap to compute, are less suitable for this specific design. For example, deep convolutional neural networks (CNNs) exhibit mathematically a very similar problem structure to seismic inverse problems, but forward and backward evaluations of CNNs are typically much faster than solving forward and adjoint wave equations, even if we consider very deep networks like ResNet [80]. Implementing training algorithms for CNNs as an event-driven workflow as presented here, is therefore excessive for the problem sizes that are currently encountered in deep learning, but might be justified in the future if the dimensionality of neural networks continues to grow.

The event-driven workflow presented in this work was specifically designed for AWS and takes advantage of specialized services for batch computing or event-driven computations that are available on this platform. However, in principle, it is possible to implement our workflow on other cloud platforms as well, as almost all of the utilized services have equivalent versions on Microsoft Azure or the Google Cloud Platform (Table 3] [81, 82]. Services for running parallel containerized workloads in the cloud, as well as event-driven cloud functions, which are the two main components of our workflow, are available on all platforms considered in our comparison. Furthermore, both Microsoft Azure as well as the GCP offer similar Python APIs as AWS for interfacing cloud services. We also speculate that, as cloud technology matures, services between different providers will likely grow more similar to each other. This is based on the presumption that less advanced cloud platforms will imitate services offered by major cloud providers in order to be competitive in the growing cloud market.

Overall, our workflow and performance evaluation demonstrate that cost-competitive HPC in the cloud is possible, but requires a fundamental software re-design of the corresponding application. In our case, the implementation of an eventdriven seismic imaging workflow was possible, as we leverage Devito for expressing and solving the underlying wave equations, which accounts for the major workload of seismic imaging. With Devito, we are able to abstract the otherwise complex implementation and performance optimization of wave equation solvers and take advantage of recent advances in automatic code generation. As Devito generates code for solving single PDEs, with the possibility of using MPI-based domain decomposition, we are not only able to leverage AWS Batch for the parallelization over source experiments, but can also take advantage of AWS Batch's multi-node functionality to shift from data to model parallelism. In contrast, many seismic imaging codes are software monoliths, in which PDE solvers are enmeshed with IO routines, parallelization and manual performance optimization. Adapting codes of this form to the cloud is fundamentally more challenging, as it is not 
easily possible to isolate individual components such as a PDE solver for a single source location, while replacing the parallelization with cloud services. This illustrates that separation of concerns and abstract user interfaces are a prerequisite for porting HPC codes to the cloud such that the codes are able to take advantage of new technologies like object storage and event-driven computations. With a domain-specific language compiler, automatic code generation, high-throughput batch computing and serverless visual algorithm definitions, our workflow represents a true vertical integration of modern programming paradimgs into a framework for HPC in the cloud.

\section{Conclusion}

Porting HPC applications to the cloud using a lift and shift approach based on virtual clusters that emulate on-premise HPC clusters, is problematic as the cloud cannot offer the same performance and reliability as conventional clusters. Applications such as seismic imaging that are computationally expensive and run for a long time, are faced with practical challenges such as cost and resilience issues, which prohibit the cloud from being widely adapted for HPC tasks. However, the cloud offers a range of new technologies such as object storage or event-driven computations, that allow to address computational challenges in HPC in novel ways. In this work, we demonstrate how to adapt these technologies to implement a workflow for seismic imaging in the cloud that does not rely on a conventional cluster, but is instead based on serverless and event-driven computations. These tools are not only necessary to make HPC in the cloud financially viable, but also to improve the resilience of workflows. The code of our application is fully redesigned and uses a variety of AWS services as building blocks for the new workflow, thus taking advantage of AWS being responsible for resilience, job scheduling, and resource allocations. Our performance analysis shows that the resulting workflow exhibits competitive performance and scalability, but most importantly minimizes idle time on EC2 instances and cost and is inherently resilient. Our example therefore demonstrates that successfully porting HPC applications to the cloud is possible, but requires to carefully adapt the corresponding codes to to the new environment. This process is heavily dependent on the specific application and involves identifying properties of the underlying scientific problem that can be exploited by new technologies available in the cloud. Most importantly, it requires that codes are modular and designed based on the principle of separation of concerns, thus making this transition possible.

\section{Acknowledgments}

This research was funded by the Georgia Research Alliance and the Georgia Institute of Technology.

\section{Appendix}

\section{Experimental setup}

Table 4 lists the dimensions of the BP 2004 model and the corresponding seismic data set that was used in our performance analysis. Both the model and data set are publicly available from the society of exploration geophysicists [83].

Table 4: Parameters of the BP 2004 velocity benchmark model and the corresponding seismic data set.

\begin{tabular}{ll}
\hline Grid dimensions & $1,911 \times 10,789$ \\
Grid spacing $[\mathrm{m}]$ & $6.25 \times 6.25$ \\
Domain size $[\mathrm{km}]$ & $11.94 \times 67.43$ \\
Number of seismic source $n_{s}$ & 1,348 \\
Propagation time [s] & 12 \\
Number of time steps & 21,889 \\
Dimensions of each $\mathbf{d}_{\mathbf{i}}$ (reshaped to 2D array) & $2,001 \times 1,201$ \\
Dominant frequency of source [Hz] & 20 \\
\hline
\end{tabular}

\section{Computational resources}

Table 5 provides an overview of the AWS EC2 instances used in our performance analysis, including their respective CPU architectures. EC2 instances of a fixed instance type (such as $r 5$ or c5n) have the same architecture for different sizes (e.g. $2 \mathrm{xlarge}, 4 \mathrm{xlarge})$, as those instances run on the same hardware.

\section{References}

[1] A. A. Valenciano. Imaging by wave-equation inversion. $\mathrm{PhD}$ thesis, Stanford University, 2008. 
Table 5: Architectures of compute instances used in our performance analysis on AWS and Optimum.

\begin{tabular}{llcc}
\hline Instance & Intel Xeon Architecture & vCPUs & RAM (GB) \\
\hline m4.4xlarge & E5-2686 v4 @ 2.30GHz & 16 & 64 \\
r5.12xlarge & Platinum 8175M @ 2.50 GHz & 48 & 384 \\
r5.24xlarge & Platinum 8175M @ 2.50 GHz & 96 & 768 \\
c5n.9xlarge & Platinum 8124M @ 3.00 GHz & 36 & 384 \\
c5n.12xlarge & Platinum 8142M @ 3.00 GHz & 72 & 768 \\
r5.metal & Platinum 8175M @ 2.50 GHz & 96 & 768 \\
Optimum & E5-2680 v2 @ 2.80GHz & 20 & 256 \\
\hline
\end{tabular}

[2] S. Dong, J. Cai, M. Guo, S. Suh, Z. Zhang, B. Wang, and Z. Li. Least-squares reverse time migration: towards true amplitude imaging and improving the resolution. In 82nd Annual International Meeting, SEG, Expanded Abstracts, pages 1-5, 2012. doi: 10.1190/segam2012-1488.1.

[3] Chong Zeng, Shuqian Dong, and Bing Wang. Least-squares reverse time migration: Inversion-based imaging toward true reflectivity. The Leading Edge, 33:962-964,966,968, 2014. doi: 10.1190/tle33090962.1.

[4] Philipp A. Witte, Mathias Louboutin, Fabio Luporini, Gerard J. Gorman, and Felix J. Herrmann. Compressive least-squares migration with on-the-fly Fourier transforms. GEOPHYSICS, 84(5):R655-R672, 2019. doi: 10.1190/ geo2018-0490.1. URL https://doi.org/10.1190/geo2018-0490.1

[5] Seismic processing and imaging. https://www.pgs.com/imaging/services/processing-and-imaging/ 2019.

[6] Exxonmobil sets record in high-performance oil and gas reservoir computing. https://corporate.exxonmobil com/en/Energy-and-environment/Tools-and-processes/Exploration-technology/ExxonMobilsets-record-in-high-performance-oil-and-gas-reservoir-computing 2019.

[7] AWS enterprise customer success stories. https://aws.amazon.com/solutions/case-studies/enterprise. 2019.

[8] Adrian Cockroft. Netflix in the cloud. In QCon San Fransisco. Netflix, 2011.

[9] Keith R Jackson, Lavanya Ramakrishnan, Krishna Muriki, Shane Canon, Shreyas Cholia, John Shalf, Harvey J Wasserman, and Nicholas J Wright. Performance analysis of high performance computing applications on the Amazon Web Services cloud. In 2010 IEEE second international conference on cloud computing technology and science, pages 159-168. IEEE, 2010.

[10] A. Iosup, S. Ostermann, M. N. Yigitbasi, R. Prodan, T. Fahringer, and D. Epema. Performance analysis of cloud computing services for many-tasks scientific computing. IEEE Transactions on Parallel and Distributed Systems, 22 (6):931-945, June 2011. ISSN 1045-9219. doi: 10.1109/TPDS.2011.66.

[11] Simson L. Garfinkel. An evaluation of Amazon's grid computing services: EC2, S3, and SQS. In Harvard Computer Science Group Technical Report TR-08-07, 2007.

[12] Jeffrey Napper and Paolo Bientinesi. Can cloud computing reach the Top500? In Proceedings of the Combined Workshops on Unconventional High Performance Computing Workshop Plus Memory Access Workshop (UCHPC$M A W^{\prime}$ 09), UCHPC-MAW '09, pages 17-20, New York, NY, USA, 2009. ACM. ISBN 978-1-60558-557-4. doi: 10.1145/1531666.1531671. URL http://doi.acm.org/10.1145/1531666.1531671

[13] Keith R Jackson, Krishna Muriki, Lavanya Ramakrishnan, Karl J Runge, and Rollin C Thomas. Performance and cost analysis of the supernova factory on the Amazon AWS cloud. Scientific Programming, 19(2-3):107-119, 2011.

[14] Lavanya Ramakrishnan, Piotr T Zbiegel, Scott Campbell, Rick Bradshaw, Richard Shane Canon, Susan Coghlan, Iwona Sakrejda, Narayan Desai, Tina Declerck, and Anping Liu. Magellan: experiences from a science cloud. In Proceedings of the 2nd international workshop on Scientific cloud computing, pages 49-58. ACM, 2011.

[15] Lavanya Ramakrishnan, R Shane Canon, Krishna Muriki, Iwona Sakrejda, and Nicholas J Wright. Evaluating interconnect and virtualization performance for high performance computing. ACM SIGMETRICS Performance Evaluation Review, 40(2):55-60, 2012.

[16] Shajulin Benedict. Performance issues and performance analysis tools for HPC cloud applications: a survey. Computing, 95(2):89-108, 2013.

[17] Piyush Mehrotra, Jahed Djomehri, Steve Heistand, Robert Hood, Haoqiang Jin, Arthur Lazanoff, Subhash Saini, and Rupak Biswas. Performance evaluation of Amazon Elastic Compute Cloud for NASA high-performance computing applications. Concurrency and Computation: Practice and Experience, 28(4):1041-1055, 2016.

[18] Abhishek Gupta and Dejan Milojicic. Evaluation of HPC applications on cloud. In 2011 Sixth Open Cirrus Summit, pages 22-26. IEEE, 2011. 
[19] I. Sadooghi, J. H. Martin, T. Li, K. Brandstatter, K. Maheshwari, T. P. P. de Lacerda Ruivo, G. Garzoglio, S. Timm, Y. Zhao, and I. Raicu. Understanding the performance and potential of cloud computing for scientific applications. IEEE Transactions on Cloud Computing, 5(2):358-371, April 2017. ISSN 2168-7161. doi: 10.1109/TCC.2015. 2404821 .

[20] Charlotte Kotas, Thomas Naughton, and Neena Imam. A comparison of Amazon Web Services and Microsoft Azure cloud platforms for high performance computing. In 2018 IEEE International Conference on Consumer Electronics (ICCE), pages 1-4. IEEE, 2018.

[21] Jack J Dongarra, Piotr Luszczek, and Antoine Petitet. The LINPACK benchmark: past, present and future. Concurrency and Computation: practice and experience, 15(9):803-820, 2003.

[22] Paul Rad, AT Chronopoulos, P Lama, Pranitha Madduri, and Cameron Loader. Benchmarking bare metal cloud servers for HPC applications. In 2015 IEEE International Conference on Cloud Computing in Emerging Markets (CCEM), pages 153-159. IEEE, 2015.

[23] Mohammad Mohammadi and Timur Bazhirov. Comparative benchmarking of cloud computing vendors with high performance LINPACK. In Proceedings of the 2nd International Conference on High Performance Compilation, Computing and Communications (HP3C-2018), pages 1-5. ACM, 2018.

[24] William Gropp, William D Gropp, Argonne Distinguished Fellow Emeritus Ewing Lusk, Ewing Lusk, and Anthony Skjellum. Using MPI: portable parallel programming with the message-passing interface, volume 1. MIT press, 1999.

[25] AWS documentation: AWS Lambda. https://aws.amazon.com/lambda/, 2019.

[26] AWS documentation: Amazon Simple Storage Service. https://docs . aws . amazon.com/AmazonS3/latest/ dev/Welcome.html, 2019.

[27] Google cloud storage. https://cloud.google.com/storage/ 2019.

[28] Michael Barton, Will Reese, John A Dickinson, Jay B Payne, Charles B Thier, and Gregory Holt. Massively scalable object storage system, April 28 2015. US Patent 9,021,137 to Rackspace US, Inc.

[29] Aaron Friedman and Angel Pizarro. Building high-throughput genomics batch workflows on AWS. https://aws . amazon.com/blogs/compute/building-high-throughput-genomics-batch-workflowson-aws-introduction-part-1-of-4/, 52017.

[30] Jeffrey Dean and Sanjay Ghemawat. MapReduce: simplified data processing on large clusters. Communications of the ACM, 51(1):107-113, 2008.

[31] AWS documentation: AWS Batch. https://aws.amazon.com/ec2/ 2019.

[32] Apache Hadoop. https://hadoop.apache.org/ 2019.

[33] AWS documentation: Amazon EMR. https://docs.aws.amazon.com/emr/latest/ManagementGuide/emroverview.html 2019.

[34] Frederic Billette and Sverre Brandsberg-Dahl. The 2004 BP velocity benchmark. In 67th Annual International Meeting, EAGE, Expanded Abstracts, page B035. EAGE, 2005.

[35] Philipp A. Witte, Mathias Louboutin, Henryk Modzelewski, Charles Jones, James Selvage, and Felix J. Herrmann. Event-driven workflows for large-scale seismic imaging in the cloud. In 89th Annual International Meeting, SEG, Expanded Abstracts, page 0, 2019.

[36] Advances in seismic imaging technology. https://www.hartenergy.com/exclusives/advances-seismicimaging-technology-177370, 2019.

[37] Albert Tarantola. Inversion of seismic reflection data in the acoustic approximation. Geophysics, 49(8):1259, 1984. doi: 10.1190/1.1441754. URL+http://dx.doi.org/10.1190/1.1441754

[38] Jean Virieux and Stephane Operto. An overview of full-waveform inversion in exploration geophysics. GEOPHYSICS, 74(6):WCC127-WCC152, November-December 2009. doi: 10.1190/1.3238367.

[39] R. Gerhard Pratt. Seismic waveform inversion in the frequency domain, part 1: Theory and verification in a physical scale model. Geophysics, 64(3):888-901, 1999. doi: 10.1190/1.1444597. URL https://doi.org/10.1190/1 1444597

[40] Bas Peters, Brendan R. Smithyman, and Felix J. Herrmann. Projection methods and applications for seismic nonlinear inverse problems with multiple constraints. GEOPHYSICS, 84(2):R251-R269, 2019. doi: 10.1190/geo2018-0192.1. URL https://doi.org/10.1190/geo2018-0192.1

[41] R. Courant, K. Friedrichs, and H. Lewy. On the partial difference equations of mathematical physics. International Business Machines (IBM) Journal of Research and Development, 11(2):215-234, March 1967. ISSN 0018-8646. doi: $10.1147 /$ rd.112.0215.

[42] Lars Ruthotto and Eldad Haber. Deep neural networks motivated by partial differential equations. CoRR, abs/1804.04272, 2018. URL/http://arxiv.org/abs/1804.04272 
[43] Andreas Griewank and Andrea Walther. Algorithm 799: Revolve: An implementation of checkpointing for the reverse or adjoint mode of computational differentiation. Association for Computing Machinery (ACM) Transactions on Mathematical Software, 26(1):19-45, March 2000. ISSN 0098-3500. doi: 10.1145/347837.347846. URL http://doi.acm.org/10.1145/347837.347846

[44] C. M. Furse. Faster than Fourier-ultra-efficient time-to-frequency domain conversions for FDTD. In Institute of Electrical and Electronics Engineers (IEEE): Antennas and Propagation Society International Symposium, volume 1, pages 536-539 vol.1, June 1998. doi: 10.1109/APS.1998.699196.

[45] R. Abdelkhalek, H. Calandra, O. Coulaud, J. Roman, and G. Latu. Fast seismic modeling and reverse time migration on a GPU cluster. In 2009 International Conference on High Performance Computing Simulation, pages 36-43, June 2009. doi: 10.1109/HPCSIM.2009.5192786.

[46] Robin M. Weiss and Jeffrey Shragge. Solving 3D anisotropic elastic wave equations on parallel GPU devices. GEOPHYSICS, 78(2):F7-F15, 2013. doi: 10.1190/geo2012-0063.1. URL https://doi .org/10.1190/geo20120063.1

[47] M. Louboutin, M. Lange, F. Luporini, N. Kukreja, P. A. Witte, F. J. Herrmann, P. Velesko, and G. J. Gorman. Devito (v3.1.0): an embedded domain-specific language for finite differences and geophysical exploration. Geoscientific Model Development, 12(3):1165-1187, 2019. doi: 10.5194/gmd-12-1165-2019. URL https://www.geoscimodel-dev.net/12/1165/2019/.

[48] William W. Symes, Dong Sun, and Marco Enriquez. From modelling to inversion: Designing a well-adapted simulator. Geophysical Prospecting, 59(5):814-833, 2011. ISSN 1365-2478. doi: 10.1111/j.1365-2478.2011.00977.x. URL $10.1111 / \mathrm{j} .1365-2478.2011 .00977 . \mathrm{x}$

[49] L. Ruthotto, E. Treister, and E. Haber. jInv-a flexible Julia package for PDE parameter estimation. SIAM Journal on Scientific Computing, 39(5):S702-S722, 2017. doi: 10.1137/16M1081063. URL https://doi.org/10.1137/ $16 \mathrm{M} 1081063$

[50] Curt Da Silva and Felix J. Herrmann. A unified 2D/3D large-scale software environment for nonlinear inverse problems. ACM Transactions on Mathematical Software, 45:7:1-7:35, 2017.

[51] Philipp A. Witte, Mathias Louboutin, Fabio Luporini, Navjot Kukreja, Michael Lange, Gerard J. Gorman, and Felix J. Herrmann. A large-scale framework for symbolic implementations of seismic inversion algorithms in Julia. Geophysics, 84:A31 - V183, 52019.

[52] AWS documentation: How spot instances work. https://docs.aws.amazon.com/AWSEC2/latest/ UserGuide/how-spot-instances-work.html 2019.

[53] Yurii Nesterov. Lectures on convex optimization, volume 137. Springer, 2018.

[54] Diederik P. Kingma and Jimmy Ba. Adam: A method for stochastic optimization. ArXiv e-prints, abs/1412.6980, 2014. URL https://arxiv.org/abs/1412.6980

[55] Reeves Fletcher and Colin M Reeves. Function minimization by conjugate gradients. The computer journal, 7(2): 149-154, 1964.

[56] Amir Beck and Marc Teboulle. A fast iterative shrinkage-thresholding algorithm for linear inverse problems. SIAM journal on imaging sciences, 2(1):183-202, 2009.

[57] AWS documentation: Amazon Elastic Compute Cloud. https://docs.aws.amazon.com/batch/latest/ userguide/what-is-batch.html 2019.

[58] Starcluster. http://star.mit.edu/cluster/, 2019.

[59] AWS High Performance Computing. https://aws.amazon.com/hpc/ 2019.

[60] AWS documentation: AWS Step Functions. https://aws.amazon.com/step-functions/ 2019.

[61] Iterating a loop using Lambda. https://docs.aws.amazon.com/step-functions/latest/dg/tutorialcreate-iterate-pattern-section.html 2018.

[62] Docker. https://www.docker.com/ 2019.

[63] Stefan Van Der Walt, S Chris Colbert, and Gael Varoquaux. The NumPy array: a structure for efficient numerical computation. Computing in Science \& Engineering, 13(2):22, 2011.

[64] AWS documentation: Amazon Simple Queue Service. https://docs.aws.amazon.com/AmazonS3/latest/ dev/Welcome.html, 2019.

[65] AWS documentation: Amazon EC2 instance types. https://aws.amazon.com/ec2/instance-types/. 2019.

[66] Fabio Luporini, Michael Lange, Mathias Louboutin, Navjot Kukreja, Jan Hückelheim, Charles Yount, Philipp A. Witte, Paul H. J. Kelly, Gerard J. Gorman, and Felix J. Herrmann. Architecture and performance of Devito, a system for automated stencil computation. To be published in ACM Transactions on Mathematical Software, 2018. URL https://arxiv.org/abs/1807.03032 
[67] David Joyner, Ondřej Čertík, Aaron Meurer, and Brian E. Granger. Open source computer algebra systems: SymPy. Association for Computing Machinery (ACM) Communications in Computer Algebra, 45(3/4):225-234, January 2012. ISSN 1932-2240. doi: 10.1145/2110170.2110185. URL http://doi .acm.org/10.1145/2110170.2110185

[68] N. Kukreja, J. Hückelheim, M. Lange, M. Louboutin, A. Walther, S. W. Funke, and G. Gorman. High-level Python abstractions for optimal checkpointing in inversion problems. ArXiv e-prints, January 2018.

[69] Josh Rad, Amr Ragab, and Aswin Damodar. Building a tightly coupled molecular dynamics workflow with multi-node parallel jobs in AWS Batch. https://aws.amazon.com/blogs/compute/building-a-tightlycoupled-molecular-dynamics-workflow-with-multi-node-parallel-jobs-in-aws-batch/, 112018.

[70] Boto 3 documentation. https://boto3.amazonaws.com/v1/documentation/api/latest/index.html\# 2019.

[71] Jorge Nocedal and Stephen Wright. Numerical optimization. Springer Science \& Business Media, 2006.

[72] Léon Bottou. Large-scale machine learning with stochastic gradient descent. In Proceedings of 15th International Conference on Computational Statistics (COMPSTAT'2010), pages 177-186. Springer, 2010.

[73] Minh Thanh Chung, Nguyen Quang-Hung, Manh-Thin Nguyen, and Nam Thoai. Using Docker in high performance computing applications. In 2016 IEEE Sixth International Conference on Communications and Electronics (ICCE), pages 52-57. IEEE, 2016.

[74] Alberto Valli and Alfio Quarteroni. Domain decomposition methods for partial differential equations. Numerical Mathematics and Scientific Computation. The Clarendon Press, Oxford University Press, New York, 1999.

[75] AWS documentation: AWS Batch - multi node parallel jobs. https://docs . aws . amazon.com/batch/latest/ userguide/multi-node-parallel-jobs.html 2019.

[76] Amazon EC2 C5 instances. https://aws.amazon.com/ec2/instance-types/c5/, 2019.

[77] Announcing accelerated scale-down of AWS Batch managed compute environments. https://aws .amazon.com/ about-aws/whats-new/2017/10/announcing-accelerated-scale-down-of-aws-batch-managedcompute-environments/, 2019.

[78] Wesley Bland, Aurelien Bouteiller, Thomas Herault, George Bosilca, and Jack Dongarra. Post-failure recovery of MPI communication capability: Design and rationale. The International Journal of High Performance Computing Applications, 27(3):244-254, 2013.

[79] Saurabh Hukerikar, Rizwan A Ashraf, and Christian Engelmann. Towards new metrics for high-performance computing resilience. In Proceedings of the 2017 Workshop on Fault-Tolerance for HPC at Extreme Scale, pages 23-30. ACM, 2017.

[80] Kaiming He, Xiangyu Zhang, Shaoqing Ren, and Jian Sun. Deep residual learning for image recognition. In Proceedings of the IEEE conference on computer vision and pattern recognition, pages 770-778, 2016.

[81] AWS to Azure services comparison. https://docs.microsoft.com/en-us/azure/architecture/awsprofessional/services 2019.

[82] Google Cloud Platform for AWS professionals. https://cloud.google.com/docs/compare/aws/, 2019.

[83] 2004 BP velocity estimation benchmark model. https://wiki.seg.org/wiki/2004_BP_velocity_ estimation_benchmark_model, 2019. 